\title{
Divergent Pathways Mediate Spine Alterations and Cell Death Induced by Amyloid- $\beta$, Wild-Type Tau, and R406W Tau
}

\author{
Christian Tackenberg and Roland Brandt \\ Department of Neurobiology, University of Osnabrück, D-49076 Osnabrück, Germany
}

\begin{abstract}
Alzheimer's disease is characterized by synaptic alterations and neurodegeneration. Histopathological hallmarks represent amyloid plaques composed of amyloid- $\beta(\mathrm{A} \beta)$ and neurofibrillary tangles containing hyperphosphorylated tau. To determine whether synaptic changes and neurodegeneration share common pathways, we established an ex vivo model using organotypic hippocampal slice cultures from amyloid precursor protein transgenic mice combined with virus-mediated expression of EGFP-tagged tau constructs. Confocal high-resolution imaging, algorithm-based evaluation of spines, and live imaging were used to determine spine changes and neurodegeneration. We report that $\mathrm{A} \beta$ but not tau induces spine loss and shifts spine shape from mushroom to stubby through a mechanism involving NMDA receptor (NMDAR), calcineurin, and GSK-3 $\beta$ activation. In contrast, $A \beta$ alone does not cause neurodegeneration but induces toxicity through phosphorylation of wild-type (wt) tau in an NMDAR-dependent pathway. We show that GSK-3 $\beta$ levels are elevated in APP transgenic cultures and that inhibiting GSK-3 $\beta$ activity or use of phosphorylation-blocking tau mutations prevented $\mathrm{A} \beta$-induced toxicity of tau. FTDP-17 tau mutants are differentially affected by $\mathrm{A} \beta$. While R406W tau shows increased toxicity in the presence of $\mathrm{A} \beta$, no change is observed with P301L tau. While blocking NMDAR activity abolishes toxicity of both wt and R406W tau, the inhibition of GSK- $3 \beta$ only protects against toxicity of wt tau but not of R406W tau induced by $\mathrm{A} \beta$. Tau aggregation does not correlate with toxicity. We propose that $\mathrm{A} \beta$-induced spine pathology and tau-dependent neurodegeneration are mediated by divergent pathways downstream of NMDAR activation and suggest that $\mathrm{A} \beta$ affects wt and R406W tau toxicity by different pathways downstream of NMDAR activity.
\end{abstract}

\section{Introduction}

Alzheimer's disease (AD) is characterized by massive neurodegeneration and altered neuronal connectivity. Histopathological hallmarks are the presence of extracellular amyloid plaques consisting of aggregated $\mathrm{A} \beta$ and intracellular neurofibrillary tangles (NFTs) containing hyperphosphorylated tau. Most of AD cases are spontaneous; however, familial AD (FAD) with an early onset also occurs. FAD cases are due to mutations in the presenilin (PS) genes 1 and 2 and in the APP gene, which increase generation of the most amyloidogenic form of $\mathrm{A} \beta, \mathrm{A} \beta_{42}$ (Steiner et al., 1999). While mutations in the tau gene have been reported in frontotemporal dementia and parkinsonism linked to chromosome 17 (FTDP-17), in which they cause tau aggregation and neurodegeneration, no tau mutations have been identified in $\mathrm{AD}$ (Shahani and Brandt, 2002). Based on this, the amyloid cascade hypothesis has

Received July 24, 2009; revised Sept. 1, 2009; accepted Sept. 7, 2009.

Funds have been provided by the Deutsche Forschungsgemeinschaft (DFG BR1192/11-1). We thank Dr. Sondra Schlesinger (Washington University School of Medicine) for the generous gift of pSinRep5 vector and helper DH (26S) DNA and Dr. Peter Davies for PHF1 antibody. We appreciate the help of Adnan Ghori and Christoph Kessler with morphological analyses and the help of Angelika Hilderink with Western blotting. We also thank Lidia Bakota for helpful suggestions on this manuscript.

Correspondence should be addressed to Prof. Dr. Roland Brandt, Department of Neurobiology, University of Osnabrück, Barbarastraße 11, D-49076 0snabrück, Germany. E-mail: brandt@biologie.uni-osnabrueck.de. DOI:10.1523/JNEUROSCI.3590-09.2009

Copyright $\odot 2009$ Society for Neuroscience 0270-6474/09/2914439-12\$15.00/0 been developed (Hardy and Selkoe, 2002), in which changes in tau are considered downstream of the $\mathrm{A} \beta$ pathology.

Several studies showed that synaptic changes and neurodegeneration are separated temporally. However, whether both share common pathways remains to be determined. Synaptic alterations as evidenced by a reduction in dendritic spine numbers and changes in spine shape are an early event in $\mathrm{AD}$ and are thought to be caused by soluble low-molecular-weight $\mathrm{A} \beta$ oligomers (Haass and Selkoe, 2007; Tackenberg et al., 2009). Tau hyperphosphorylation and the formation of NFTs occur later. While the amount of NFTs correlates with the degree of dementia (Braak and Braak, 1991), little is known whether and how tau affects spines and how tau and $\mathrm{A} \beta$ interfere. Rapoport et al. (2002) provided evidence for an essential role of tau in $\mathrm{A} \beta$-induced cell death. Interestingly, a hyperphosphorylationmimicking tau mutant induced massive degeneration by itself when overexpressed in neurons (Fath et al., 2002; Shahani et al., 2006), suggesting that the extent of phosphorylation determines tau toxicity. In agreement with this, $\mathrm{A} \beta$ can induce tau phosphorylation at disease-relevant sites (Ferreira et al., 1997; Zheng et al., 2002; Leschik et al., 2007). It has been shown that FTDP-17 tau mutants can induce neurodegeneration in vivo (Lewis et al., 2000; Miyasaka et al., 2005; Santacruz et al., 2005). However, the mechanism by which the toxicity of wild-type (wt) tau and FTDP-17 tau mutants is induced and whether they share a common pathway remain to be shown. 
To dissect the role of $\mathrm{A} \beta$ and tau on spine changes and cell death and to determine the mechanisms and signaling pathways involved in both processes, we established an exvivo model based on organotypic hippocampal slice cultures. Cultures were prepared from transgenic mice expressing mutated $\mathrm{APP}\left(\mathrm{APP}_{\mathrm{SDL}}\right)$ in combination with virus-mediated expression of EGFP-coupled wt tau and FTDP-17 mutated tau constructs. Confocal highresolution imaging and algorithm-based evaluation were used to determine effects on spines. Cell death was visualized by live imaging.

Our data indicate that spine changes and wt tau-mediated cell death are both caused by $\mathrm{A} \beta$ through NMDA glutamate receptors (NMDARs) and GSK-3 $\beta$ activation but involve different pathways. Furthermore, we show that the FTDP-17 mutant R406W tau is also affected by $\mathrm{A} \beta$ but in a GSK-3 $\beta$-independent pathway.

\section{Materials and Methods}

Animals. Heterozygous $\mathrm{APP}_{\mathrm{SDL}}$ transgenic C57BL/6 mice (Aventis Pharma) and nontransgenic littermates (C57BL/6 mice; Charles River Laboratories and Harlan Winkelmann) were used. $\mathrm{APP}_{\mathrm{SDL}}$ transgenic mice express human APP695 with three familial Alzheimer's disease mutations in one construct, the Swedish (KM595/596NL), Dutch (E618Q), and London (V642I) mutations, under the control of the platelet-derived growth factor $\beta$ promoter (Blanchard et al., 2003). All animals were maintained and killed according to National Institutes of Health guidelines and German animal care regulations. Genotyping was performed by PCR from DNA extracted from mouse tail using the following primers: APP-forward, 5'-GTAGCAGAGGAGGAAGAAGTG-3'; and APPreverse, $5^{\prime}$-CATGACCTGGGACATTCTC- $3^{\prime}$. Primers were purchased from Biomers.

Materials. Chemicals were purchased from Sigma. Culture medium and supplements were obtained from Sigma and Invitrogen, culture dishes and plates from Nunc, and membrane culture inserts from Millipore. Gamma-secretase inhibitor $N$-[N-(3,5-difluorophenacetyl)-1-alanyl]-Sphenylglycine $t$-butyl ester (DAPT) was purchased from Merck.

Sindbis virus constructs. Construction of virus was performed as described previously (Shahani et al., 2006). The following virus constructs were used for experiments: pSinRep5-EGFP, pSinRep5-EGFP-352wt tau, pSinRep5-EGFP-352 pseudohyperphosphorylated (PHP) tau, pSinRep5EGFP-352 Ala tau, pSinRep5-EGFP-441 R406W tau, and pSinRep5-EGFP441 P301L tau. For PHP tau and Ala tau, 10 sites (Ser198, Ser199, Ser202, Thr231, Ser235, Ser396, Ser404, Ser409, Ser413, and Ser422) were mutated to glutamate and alanine, respectively (Eidenmüller et al., 2001).

Organotypic hippocampal slice cultures and Sindbis virus infection. Organotypic hippocampal slice cultures were prepared and cultured according to the study by Stoppini et al. (1991). In short, 6- to 7-d-old APP transgenic and nontransgenic C57BL/6 mice were decapitated, brains were removed, and both hippocampi were isolated and cut into $400-\mu \mathrm{m}$ thick slices by use of a McIllwain tissue chopper (Gabler). Slices were cultured on Millicell culture plate inserts $(0.4 \mu \mathrm{m}$, Millipore) in six-well plates containing $1 \mathrm{ml}$ of culture medium ( $46 \%$ minimum essential medium Eagle with HEPES modification, 25\% basal medium with Earle's modification, $25 \%$ heat-inactivated horse serum, 2 mm glutamine, $0.6 \%$ glucose, $\mathrm{pH} 7.2$ ). Culture plates were kept at $37^{\circ} \mathrm{C}$ in a humidified atmosphere containing $5 \% \mathrm{CO}_{2}$. Slices were kept in culture for $12 \mathrm{~d}$ before the experiments. Culture medium was exchanged every second or third day. On day 11 culture medium was replaced by low-serum $\mathrm{Nb}-\mathrm{N} 1$ medium (94.5\% neurobasal medium, $0.5 \%$ heat-inactivated horse serum, $2 \mathrm{~mm}$ glutamine, $0.6 \%$ glucose, $1 \times \mathrm{N} 1$ supplement, $\mathrm{pH} 7.2)$. On day 12 in vitro slice cultures were infected with Sindbis virus using a droplet method (Shahani et al., 2006). For live imaging, culture plate inserts were transferred from six-well plates into glass-bottom dishes (MatTek). For spine analysis, cultures were fixed at day 3 postinfection within six-well plates. Slices were left attached to the culture plate membrane to preserve hippocampal structure and rinsed with PBS. Slices were then fixed with $4 \%$ paraformaldehyde in PBS containing $4 \%$ sucrose for $2 \mathrm{~h}$ at $4^{\circ} \mathrm{C}$. After being washed with PBS, cultures were mounted with Confocal matrix
(Micro-Tech-Lab) and coverslipped. To compare the expression levels of EGFP-coupled tau constructs, the fluorescence intensity of infected neurons was determined. Hippocampal slice cultures were infected with the respective construct and fixed on day 3 postinfection. Confocal $z$-stack images were acquired using identical microscope settings for the respective constructs. Using the ImageJ program, all optical sections were scaled to 32-bit format and summed up. It was verified by software tools that no pixels were saturated. Integrated fluorescence intensities were determined relative to background fluorescence for 10 neurons per construct.

Treatment of hippocampal slice cultures. The following inhibitors were used to treat hippocampal slice cultures at the indicated concentrations (in $\mu \mathrm{M}$ ): 0.5 and $1 \gamma$-secretase inhibitor DAPT, 20 NMDAR antagonist 3-(2-carboxypiperazin-4-yl)propyl-1-phosphonic acid (CPP), 1 calcineurin inhibitor tacrolimus (FK-506), and 10 GSK-3 $\beta$ inhibitor 4-benzyl-2-methyl-1,2,4-thiadiazolidine-3,5-dione (TDZD). For analyses of effects on spines, all media were continuously supplemented with the respective inhibitor. For assessment of cell death, inhibitors were added only after replacing the culture medium with Nb-N1.

Antibodies. The following primary antibodies were used: phosphorylationindependent tau antibody Tau-5 (mouse; PharMingen), phosphorylationdependent tau antibody PHF-1 (mouse; a generous gift from Peter Davies, Albert Einstein College of Medicine, Bronx, NY), anti-synaptophysin (mouse; Millipore), tubulin antibody DM1A (mouse; Sigma), and anti-GSK- $3 \beta$ and phospho-GSK- $3 \alpha / \beta$ (mouse; Cell Signaling Technology). As secondary antibodies, cyanine 3 (Cy3)-coupled anti-mouse antibody (Dianova) and peroxidase-conjugated anti-mouse antibodies (Jackson ImmunoResearch) were used.

Immunohistochemistry. Infected hippocampal slices were left attached on the insert membranes throughout the immunostaining protocol to preserve the hippocampal structure. Immunostaining was performed free floating to ensure penetration of antibodies inside the tissue. Slices were first washed with PBS and fixed with $4 \%$ paraformaldehyde in PBS containing $4 \%$ sucrose for $2 \mathrm{~h}$ at $4^{\circ} \mathrm{C}$. After washing with PBS, slices were treated with $1 \%$ Triton X-100 in PBS for 90 min and 50 mm ammonium chloride for $45 \mathrm{~min}$ at room temperature. Slices were blocked with PBS containing $5 \%$ fetal calf serum, $1 \%$ BSA, and $0.1 \%$ Triton X-100 at $4^{\circ} \mathrm{C}$ overnight followed by incubation for $5 \mathrm{~d}$ at $4^{\circ} \mathrm{C}$ with primary antibodies diluted in blocking solution. After being washed with PBS, slices were incubated with Cy3-coupled anti-mouse antibody for $3 \mathrm{~d}$ at $4^{\circ} \mathrm{C}$. The slices were then washed in PBS, mounted in Confocal-Matrix (MicroTech-Lab), and coverslipped.

Immunoblot analysis. Cultured hippocampal slices were harvested on day 15, sonicated in RIPA buffer ( $50 \mathrm{~mm}$ Tris- $\mathrm{HCl}, 150 \mathrm{~mm} \mathrm{NaCl}, 2 \mathrm{~mm}$ EDTA, $1 \%$ NP- $40,0.5 \%$ deoxycholate, and $0.1 \%$ SDS, pH 8.0) containing protease inhibitors ( $1 \mathrm{~mm}$ PMSF, $10 \mu \mathrm{g} / \mathrm{ml}$ each of leupeptin and pepstatin, $1 \mathrm{~mm}$ EGTA) and phosphatase inhibitors (1 $\mathrm{mm}$ sodium orthovanadate, $20 \mathrm{~mm}$ sodium fluoride, and $1 \mathrm{~mm}$ sodium pyrophosphate), and centrifuged for $15 \mathrm{~min}$ at $13,000 \times g$ at $4^{\circ} \mathrm{C}$. The supernatant (lysate) was collected, frozen, and stored at $-80^{\circ} \mathrm{C}$. Twenty percent of lysates were subjected to SDS-PAGE and transferred to Immobilon-P (Millipore) followed by immunoblotting with various antibodies. As secondary antibody, peroxidase-coupled anti-mouse antiserum was used. Detection used enhanced chemiluminescence using SuperSignal West Dura extended-duration substrate (Pierce) and was performed according to the manufacturer's protocol. Blots were quantified with Gel-Pro Analyzer 4.0 (Media Cybernetics).

Sequential tau extraction. Tau solubility profiles were generated as previously described (Shahani et al., 2006) but without sonication, using the following buffers: (1) high-salt buffer ( $750 \mathrm{~mm} \mathrm{NaCl}, 50 \mathrm{~mm}$ Tris buffer, pH 7.4), (2) $1 \%$ Triton in high-salt buffer, (3) RIPA buffer, (4) $2 \%$ SDS, and (5) $70 \%$ formic acid (FA). All buffers were supplemented with phosphatase and protease inhibitors as described above. The same amounts of extracts $(20 \%)$ were loaded per lane and stained with Tau- 5 antibody to detect total tau.

Confocal live imaging and assessment of cell death. All images were acquired on inverted Nikon confocal laser scanning microscope Eclipse TE2000-U using argon laser (488 nm). Microscope was equipped with an incubation chamber (Solent Scientific) generating a $37^{\circ} \mathrm{C}$ humidified 

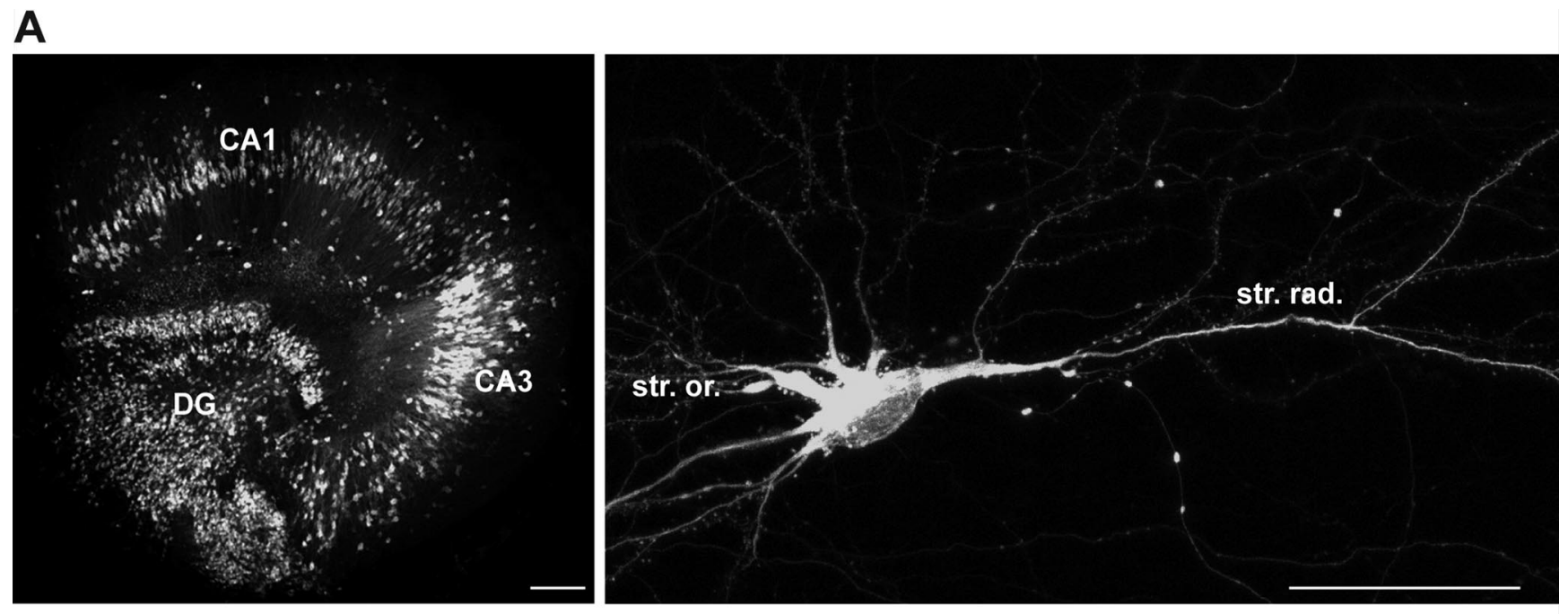

B
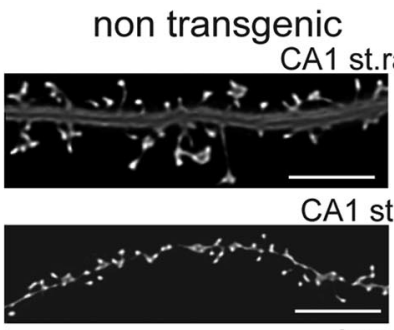

CA1

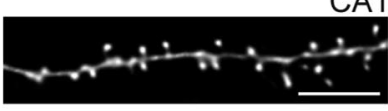

APP transgenic

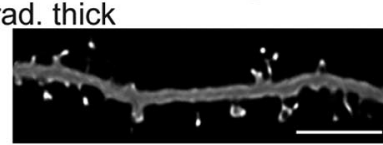

.rad. thin

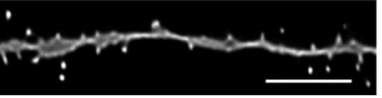

st.or.

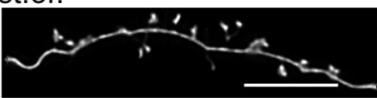

non transgenic

CA3 st.rad. thick

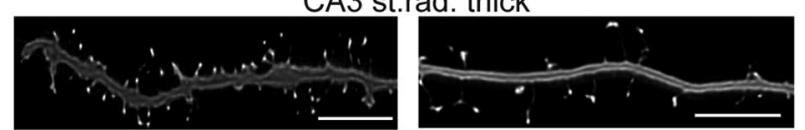

CA3 st.rad. thin

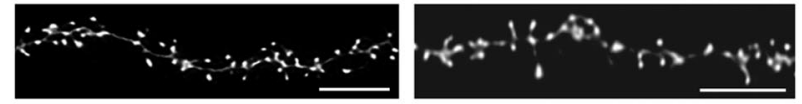

CA3 st.or.
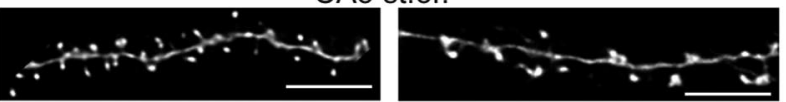

C

$$
\begin{aligned}
& \square \text { non transgenic } \\
& \square \text { APP transgenic }
\end{aligned}
$$

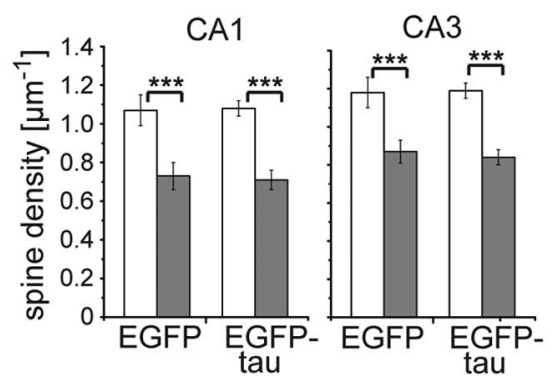

D

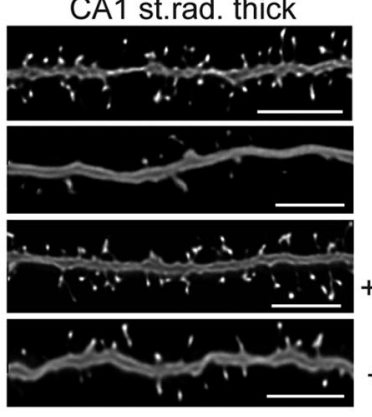

non transgenic

APP transgenic

\section{APP} $+0,5 \mu \mathrm{M}$ DAPT $\begin{aligned} \text { APP } & \\ +1 \mu \mathrm{M} \text { DAPT } & \end{aligned}$

Figure 1. Spine density of EGFP- and EGFP-tau-expressing neurons in hippocampal slice cultures. $A$, Confocal image of a whole slice (left; scale bar, $300 \mu \mathrm{m}$ ) after Sindbis virus-mediated expression of EGFP-tau. Note that tau is expressed in every hippocampal subregion with highest efficiency in CA3. Typical morphology of a CA3 pyramidal neuron (right; scale bar, $25 \mu \mathrm{m}$ ) with basal dendrites in stratum oriens and apical dendrites in stratum radiatum region of the hippocampus. $\boldsymbol{B}$, Representative high-resolution images of 20 - to 30 - $\mu \mathrm{m}$-long dendritic fragments of stratum radiatum thick and thin and stratum oriens from CA1 and CA3 neurons after blind deconvolution. Scale bar, $5 \mu \mathrm{m}$. C, Spine density in hippocampal CA1 and CA3 neurons from APP transgenic and nontransgenic mice after targeted expression of EGFP or EGFP-tau $[n=19$ (EGFP), $n=10$ (EGFP-tau)]. Spine density is strongly reduced on APP transgenic background independent of the presence of tau. $\boldsymbol{D}$, Effect of $\gamma$-secretase inhibitor DAPT on spine density of EGFP-tau-expressing neurons from APP transgenic and nontransgenic mice. High-resolution image from CA1 stratum radiatum thick (left; scale bar, $5 \mu \mathrm{m}$ ). For quantitative analysis of spine density (right), data from CA1 and CA3 regions were pooled. DAPT $(0.5 \mu \mathrm{m})$ completely abolished spine loss, whereas $1 \mu \mathrm{m}$ DAPT had only a partial albeit still significant effect ( $n=41$ for $0.5 \mu \mathrm{m} \mathrm{DAPT}$ treatment and $n=40$ for $1 \mu \mathrm{m} \mathrm{DAPT-treated} \mathrm{and} \mathrm{untreated} \mathrm{cultures).} \mathrm{Analysis} \mathrm{of} \mathrm{spine} \mathrm{density} \mathrm{shows} \mathrm{a} \mathrm{reduction} \mathrm{of} \mathrm{spine} \mathrm{loss} \mathrm{by} \mathrm{DAPT,}$ which is maximal at $0.5 \mu \mathrm{M}$. All values are shown as mean \pm SEM $\left({ }^{* *} p<0.01\right.$, ${ }^{* * *} p<0.001$; one-tailed unpaired Student's $t$ test). DG, Dentate gyrus; str.or., stratum oriens; str.rad., stratum radiatum; non tg., nontransgenic.

atmosphere containing $5 \% \mathrm{CO}_{2}$ to avoid neuronal death caused by low temperature or $\mathrm{pH}$ shifts. Objectives used for live imaging were $4 \times$ (dry, NA: 0.13 ) and $20 \times$ [dry, ELWD (extra long working distance), NA: 0.45]. Live imaging of infected hippocampal CA3 pyramidal neurons was performed over a period of $2-4 \mathrm{~d}$ postinfection. Image stacks were taken at $1024 \times 1024$ pixels with pixel size of $0.62 \mu \mathrm{m}$ in the $x$ and $y$ directions and $2.55 \mu \mathrm{m}$ steps in the $z$ direction. The lowest laser intensity and pixel dwell time possible were used to reduce phototoxicity. Maximumintensity projections of $z$ sections were created using Nikon EZ-C1 3.0 software. For analysis of cell death the number of nondegenerated neurons was counted on days 2, 3, and 4 for APP transgenic slices or nontransgenic controls expressing different EGFP-coupled tau constructs. Percentages of remaining neurons on days 3 and 4 were presented based on the respective nontransgenic control (see Fig. 4) or relative to the number of neurons at day 2 for the respective expressed construct (see Fig. 5). Nondegenerated neurons were considered to have processes without any varicosities and an intact cell body without any swellings. For quantification, only pyramidal neurons with clear triangular shape 
A

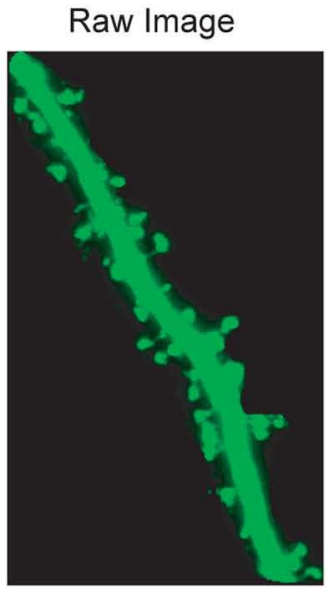

B

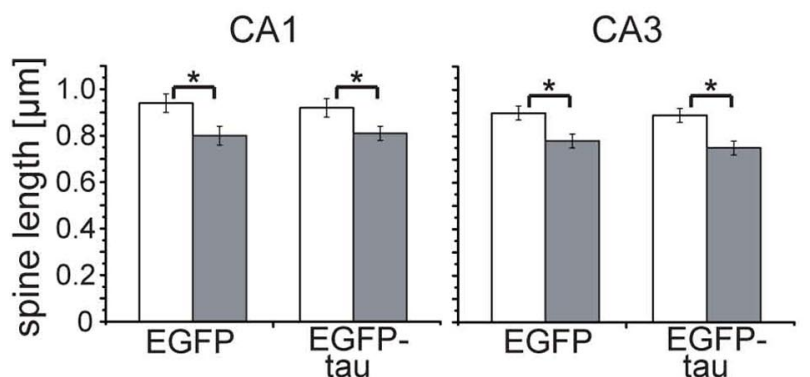

Deconvolution

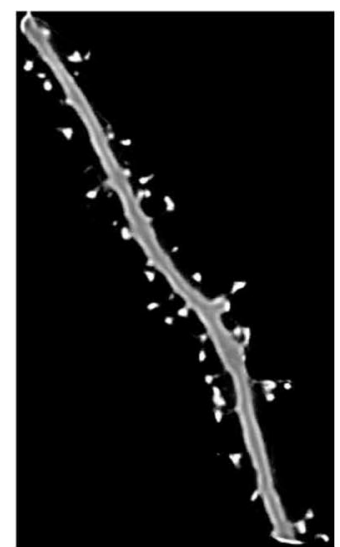

Medial axis extraction

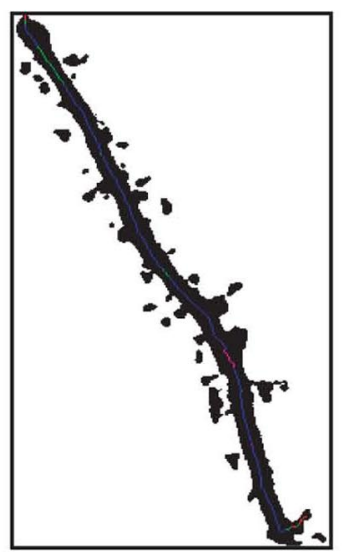

Spine detection

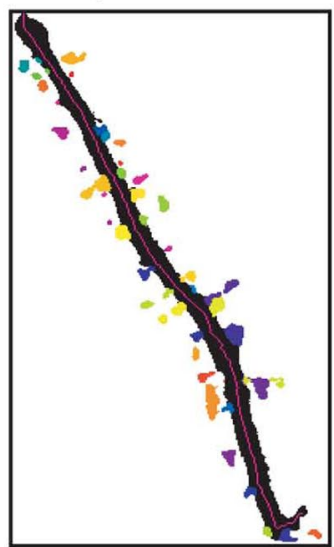

C

$\square$ non transgenic $\square$ APP transgenic

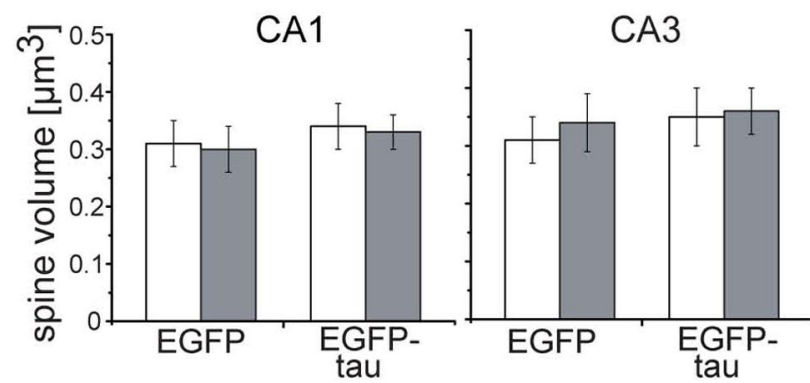

D

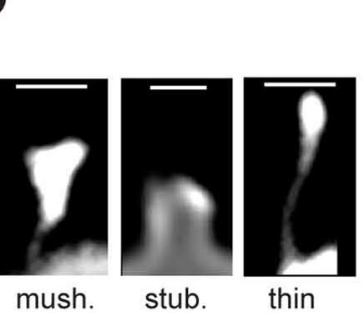

E

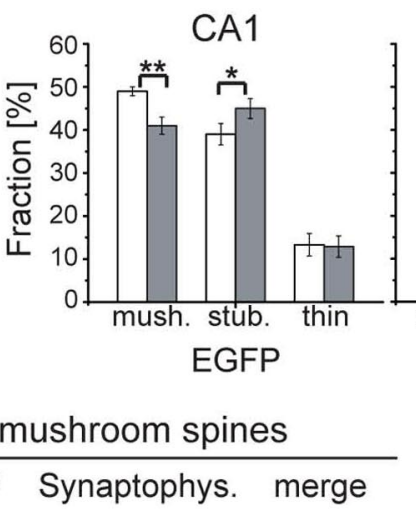

non transgenic
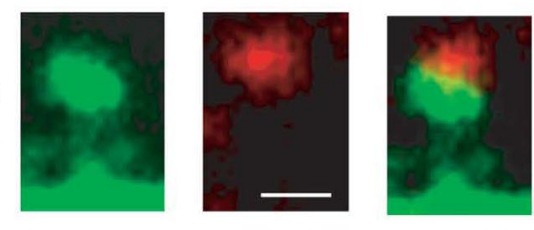

APP transgenic
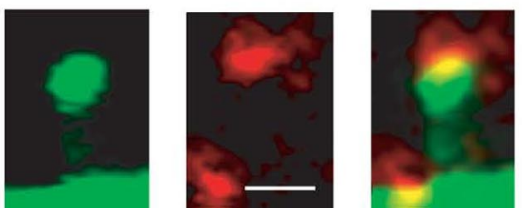

CA1

CA3

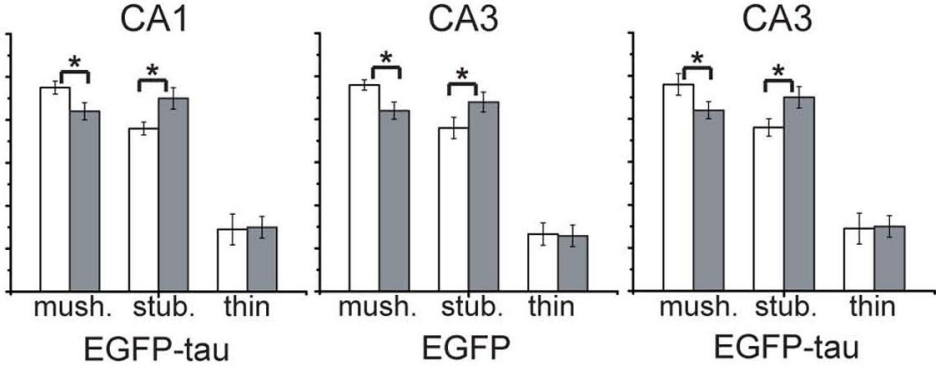

EGFP-tau

stubby spines
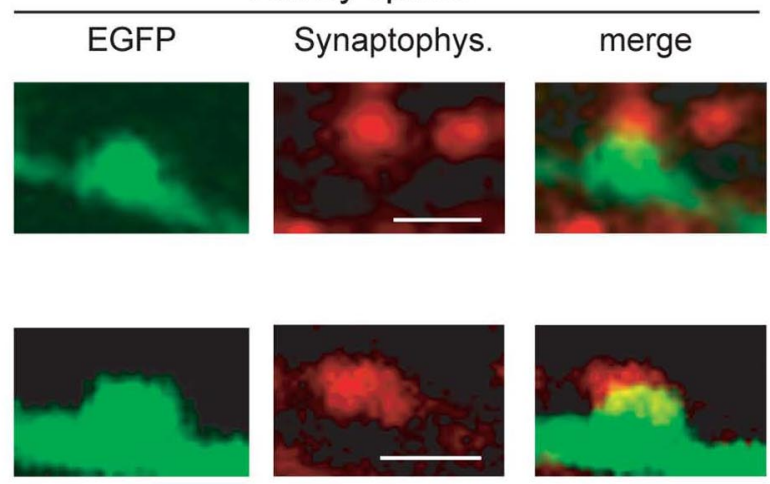

Figure 2. Spine morphology of EGFP- and EGFP-tau-expressing neurons in hippocampal slice cultures. $A$, Steps of image processing for detection and analysis of spines. Confocal raw image was deconvoluted using 3D blind algorithm (Autodeblur). Medial axis extraction was performed by software 3DMA neuron for identification of dendritic backbone. Spine detection routine allowed determining single spines, which were automatically analyzed for length and volume. Spine shape was classified by 3DMA neuron software to one of the following three types: "mushroom," "stubby," or "thin." B, Spine length in hippocampal CA1 and CA3 pyramidal neurons from APP transgenic and nontransgenic mice after targeted expression of EGFP or EGFP-tau. Spine length is significantly reduced on APP transgenic background independent of tau. C, Spine volume in neurons from APP transgenic and nontransgenic mice after targeted expression of EGFP or EGFP-tau. No difference is observed in spine volume between APP transgenic and nontransgenic cultures. D, Fraction of spines with different shape. Representative high-resolution images (left) show the three different spine types for classification, namely, mushroom (left), stubby (middle), and thin (right). The fraction of mushroom spines is significantly decreased, while stubby spines increase in CA1 and CA3 neurons from APP transgenic mice, independent of the presence of tau. $\boldsymbol{E}$, Representative images of EGFP-labeled postsynaptic spines with (Figure legend continues.) 
and apical and basal dendrites were counted, whereas interneurons were omitted. Infected CA3 regions had to have at least 15 or more infected neurons to be taken into evaluation to avoid any bias due to low sample number. Slices with a fewer number of neurons were disregarded.

Confocal imaging of fixed hippocampal slices and analysis of spine density. Confocal high-resolution imaging of spines was performed using Nikon confocal laser scanning microscope Eclipse TE2000-U with a $60 \times$ objective (oil, NA: 1.4). Twenty- to 30- $\mu$ m-long fragments of different dendritic subregions (stratum oriens and the proximal and medial part of stratum radiatum thick and thin) of hippocampal CA1 and CA3 pyramidal neurons were imaged with voxel size of $0.08 \times 0.08 \times 0.25 \mu \mathrm{m}$ in the $x-y-z$ directions. Image size was adjusted according to the length and shape of the imaged dendritic fragment. Image stacks were further processed as described for morphological spine analysis. To determine spine density, maximum projections were analyzed using NIH ImageJ software. The length of the dendrite was measured, and spines were counted as protrusions in the $x$ and $y$ axes. In addition, to visualize potential colocalizations of postsynaptic spines with presynaptic boutons, some slices were stained against synaptophysin. Images were recorded by sequential scanning using argon and helium-neon laser (544 nm).

Image processing and semiautomated analysis of spine morphology. Image stacks (Nikon .ids files) were processed using 3D blind deconvolution (10-15 iterations, Autodeblur Software) to improve signal-noise ratio and spatial resolution. Analysis of spine length, volume, and shape was performed using 3DMA neuron software (Koh et al., 2002) which allows algorithm-based, semiautomated evaluation of spine morphology.

Statistical analysis. For analysis of spine data or live imaging, statistical evaluation was performed using a one-tailed, unpaired Student's $t$ test. Data are shown as mean \pm SEM. For spine analysis, $n$ is the number of different analyzed images from at least four mice. For live imaging, $n$ is the number of analyzed hippocampal slices from at least four mice. For statistical evaluation of Western blots, experiments were performed in triplicate. Data are shown as mean $\pm \mathrm{SD}$. Statistical analysis was performed using paired Student's $t$ test. $p$ values are as follows: ${ }^{*} p<0.05$, ${ }^{* *} p<0.01$, and ${ }^{* *} p<0.001$.

\section{Results}

\section{A $\beta$ but not tau induces spine loss in hippocampal neurons}

To determine the functional interaction of tau and $A \beta$, EGFPtagged tau constructs were expressed in organotypic hippocampal slices prepared from APP transgenic and nontransgenic mice. For the experiments, Sindbis viral vectors, which allow a targeted transient expression in different types of neurons, were used (Ehrengruber et al., 1999; Shahani et al., 2006). Efficient infection of neurons in all regions of the hippocampal slices was observed from 2 to $5 \mathrm{~d}$ postinfection as evidenced by the intense EGFP fluorescence (Fig. 1A, left). At higher magnification, single CA1 and CA3 neurons could be imaged and individual spines in different layers and dendritic segments visualized (Fig. $1 A$, right).

To determine differences in spine densities, dendritic segments of stratum radiatum thick and thin and stratum oriens of EGFP-tau-expressing CA1 and CA3 neurons were imaged at high resolution. In most segments, spine number appeared to be reduced on an APP background compared with the nontransgenic control (Fig. $1 B$ ). No difference was seen in CA1 stratum oriens and CA3 stratum radiatum thin. Quantification of spine densities confirmed that spine number was significantly reduced $(p<0.001)$ in CA1 and CA3 neurons from APP transgenic mice (Fig. 1C). To determine a potential effect of tau on spine number, neurons expressing EGFP-tau or EGFP

$\leftarrow$

(Figure legend continued.) synaptophysin-positive presynaptic boutons. All values are shown as mean \pm SEM ( ${ }^{*} p<0.05 ;{ }^{* *} p<0.01$; one-tailed unpaired Student's $t$ test). $n=19$ (EGFP), $n=20$ (EGFP-tau). mush., Mushroom spine; stub., stubby spine; Synaptophys., synaptophysin. Scale bars, $0.5 \mu \mathrm{m}$. alone were compared. We did not observe any difference in spine densities. This indicates that tau does not affect the number of spines at our conditions.

To test whether spine number reduction was caused by mutated APP or by A $\beta$, the nontransition state $\gamma$-secretase inhibitor DAPT was added to the cultures to block $A \beta$ generation (Dovey et al., 2001). The presence of DAPT reduced spine loss in slices from APP transgenic mice (Fig. $1 D$ ). Interestingly, $0.5 \mu \mathrm{M}$ DAPT completely abolished spine loss $(p<0.001)$, whereas $1 \mu \mathrm{M}$ DAPT had only a partial albeit still significant effect $(p<0.001)$, indicating that the presence of $A \beta$ and not APP is responsible for spine loss. It should however be noted that we cannot completely exclude that also the APP intracellular domain (AICD) is involved in pathologic processes, since DAPT treatment also prevents AICD production.

The fact that higher concentrations of DAPT were less effective may be due to increased toxicity of DAPT at $1 \mu \mathrm{M}$, which was evident by an increased loss of neurons (data not shown).

\section{Length is reduced and shape is changed in spines from APP transgenic cultures}

Evidence exists that memory formation results in structural plasticity as seen in changes in the shape of spines (Bourne and Harris, 2007). To determine whether $A \beta$ induces alterations in spine shape of EGFP- and EGFP-tau-expressing neurons, a computerassisted method was used which permits a semiautomated detection combined with a measurement of length and volume of individual spines (Fig. 2A). Spine length was significantly reduced by $\sim 15 \%$ in APP transgenic slices in CA1 and CA3 neurons (Fig. $2 B$ ). In contrast, no difference was observed in spine volume between APP transgenic and nontransgenic cultures (Fig. $2 C)$. Interestingly, spine length was also reduced in regions in which no loss of spines was observed (e.g., CA3 stratum radiatum thin), indicating that $\mathrm{A} \beta$ does not induce a selective loss of long spines but generally reduces spine lengths (data not shown).

For further characterization of spine changes, spines were classified into the three categories, namely, "mushroom," "stubby," and "thin" (Peters and Kaiserman-Abramof, 1970), by using an algorithm-based computer-assisted method (Fig. 2D). In the CA1 and CA3 regions, the fraction of mushroom spines significantly decreased, while stubby spines increased. Again, changes were also observed in dendritic subregions in which no loss of spines occurred, suggesting that mushroom spines do not vanish but change to stubby shape. Importantly, no difference in spine morphology was observed between EGFP- and EGFP-tau-expressing neurons, confirming that tau does not affect spines.

To ascertain whether the remaining spines bear synapses, we stained slices with an antibody against synaptophysin to label presynaptic boutons (Fig. 2E). Mushroom as well as stubby spines from nontransgenic and APP transgenic animals were in close apposition with synaptophysin dots, suggesting the presence of functional synapses also after the change in spine shape.

\section{Inhibition of NMDARs, calcineurin, or GSK-3 $\beta$ abolishes $\mathrm{A} \boldsymbol{\beta}$-induced spine alterations}

It has been shown that $\mathrm{A} \beta$ can bind to NMDARs (Lacor et al., 2007 ) and that blockage of NMDAR activity reduces spine loss that had been induced by addition of soluble $\mathrm{A} \beta$ oligomers from AD patients to slice cultures (Shankar et al., 2007). To determine whether NMDARs are involved in $\mathrm{A} \beta$-mediated spine loss and morphological changes, cultures from APP transgenic and nontransgenic mice were treated with the NMDAR antagonist CPP. We observed that CPP abolished the reduction in spine density in 
cultures from APP transgenic mice (Fig. $3 A)$. Interestingly, CPP slightly but significantly reduced spine density in nontransgenic controls by $\sim 10 \%$, while it strongly increased spine density in APP transgenic cultures $(p<0.001)$ to control levels. CPP also abolished the difference between spine length of APP transgenic and nontransgenic animals (Fig. 3B). However, in this case, this was due to a reduction of spine length in control cultures rather than to an increase in APP transgenic cultures. We did not observe any influence of CPP on the volume of the spines (Fig. $3 B$ ). This is in agreement with our finding that $\mathrm{A} \beta$ did not affect spine volume (see above). Analysis of spine shape showed no effect of CPP on the fraction of the three spine types in nontransgenic controls (Fig. 3C). In APP transgenic cultures CPP treatment increased the fraction of mushroom-shaped spines and decreased stubby spines to control levels. The increase in mushroom-shaped spines reached significance in CA3 neurons $(p=0.02)$.

In addition to NMDAR activity, calcineurin is thought to be involved in $A \beta$ mediated spine loss (Shankar et al., 2007). Blocking calcineurin activity with tacrolimus (FK-506) increased spine density in APP transgenic slices but reduced spine density in controls (Fig. 3D) closely resembling the effect of CPP. This suggests that calcineurin is acting downstream of NMDAR activation. Recently it has been shown that $A \beta$ induces long-term depression (LTD) via activation of calcineurin and GSK-3 $\beta$ (Li et al., 2009). To determine whether active GSK-3 $\beta$ is also involved in $\mathrm{A} \beta$-mediated spine loss, we treated the cultures with the GSK-3 $\beta$ inhibitor TDZD (Chen et al., 2007). TDZD completely abolished $\mathrm{A} \beta$-induced spine loss in the absence of any side effects on control cultures (Fig. 3E), suggesting that GSK- $3 \beta$ operates downstream of calcineurin. Previously it has been shown that during LTD calcineurin activates protein phosphatase 1 (PP1), which in turn activates GSK-3 $\beta$ (Mulkey et al., 1994; Peineau et al., 2007) confirming the presence of this pathway.

\section{A $\beta$ induces wt tau toxicity by a pathway involving NMDAR and GSK- $3 \boldsymbol{\beta}$ but not calcineurin} Evidence exists that both $\mathrm{A} \beta$ and tau contribute to the loss of neurons observed in AD (Rapoport et al., 2002). To analyze a potential functional interaction between tau and $\mathrm{A} \beta$, organotypic hippocampal slices from transgenic and nontransgenic animals were infected with virus expressing EGFP-wt tau or EGFP alone. Cell survival was analyzed by live imaging of neurons in the CA3 region. The CA3

A

B

E
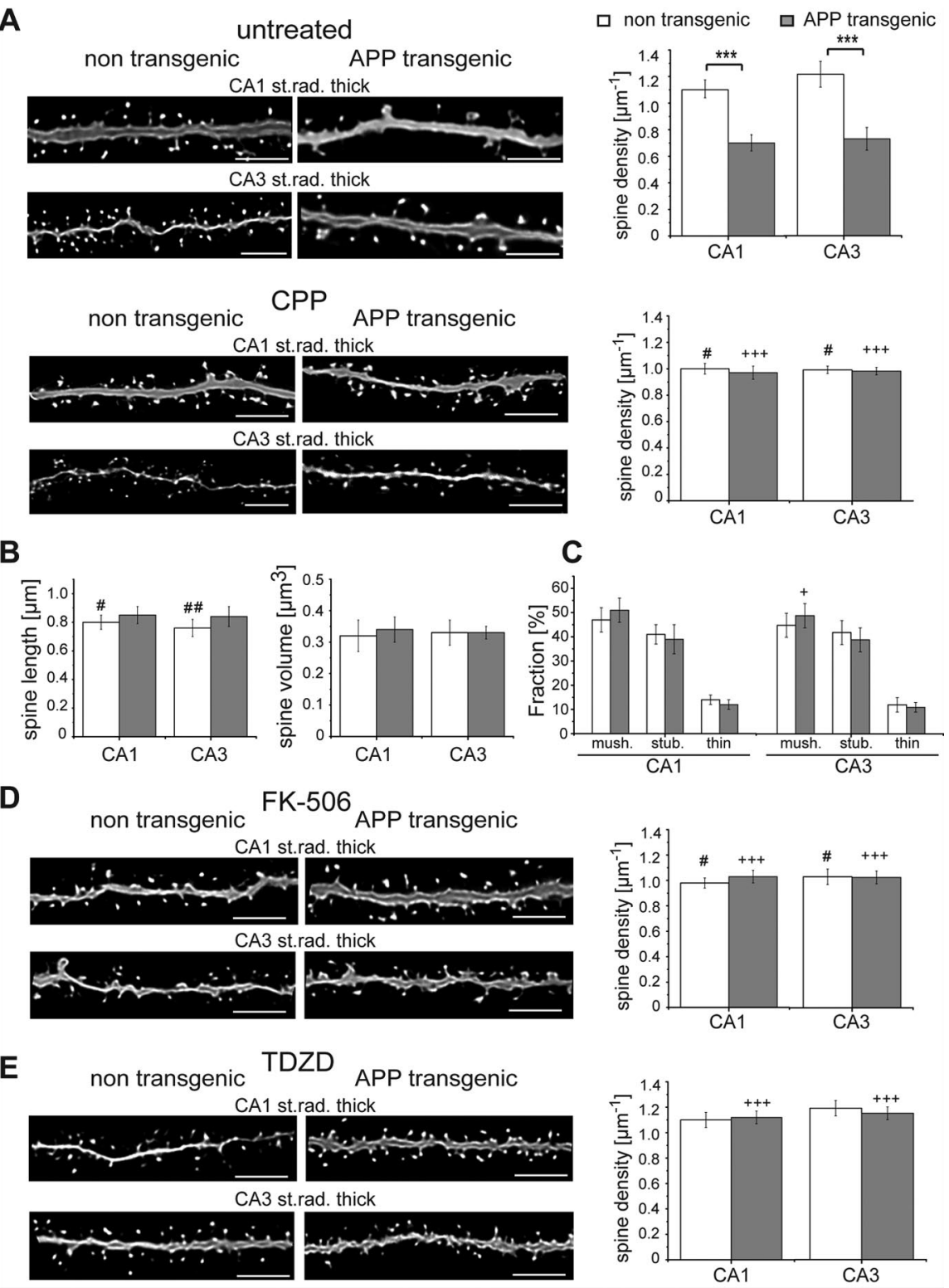

Figure 3. Effect of NMDAR antagonist CPP, calcineurin inhibitor FK-506, and GSK-3 $\beta$ inhibitor TDZD on spines in hippocampal slice cultures. $A$, Spine density in EGFP-expressing hippocampal CA1 and CA3 neurons from APP transgenic and nontransgenic mice without treatment (top) and after treatment with $20 \mu \mathrm{M}$ CPP (bottom). Representative high-resolution images of 20- to 30- $\mu \mathrm{m}$ long dendritic fragments of stratum radiatum thick from $C A 1$ and $C A 3$ neurons after blind deconvolution (left) and quantification of spine density (right) are shown. In untreated slices from APP transgenic mice, spine density is strongly reduced compared with nontransgenic slices $(n=10)$. After (PP treatment, spine density does not differ between APP transgenic and nontransgenic slices $[n=18$ (nontransgenic), $n=17$ (APP transgenic)]. Compared with untreated cultures (Fig. 10) spine density is reduced on nontransgenic background and increased for APP transgenic mice. B, Spine length and volume after treatment with $20 \mu \mathrm{M}$ CPP. No difference in spine length is observed between APP transgenic and nontransgenic controls after CPP treatment. Compared with untreated cultures, spine length is significantly reduced in controls. CPP has no effect on spine volume. C, Fraction of spines with different shapes after CPP treatment. CPP increases the fraction of mushroom-shaped spines in APP transgenic cultures to control levels. $\boldsymbol{D}$, Representative images of dendritic fragments after treatment with $1 \mu \mathrm{m} \mathrm{FK-506} \mathrm{(left)} \mathrm{and} \mathrm{quantification} \mathrm{of} \mathrm{spine} \mathrm{density}$ (right). FK-506 increases spine density in APP transgenic slices while reducing spine density in controls $(n=14)$. $\boldsymbol{E}$, Representative images of dendritic fragments after treatment with $10 \mu \mathrm{M}$ TDZD (left) and quantification of spine density (right). TDZD completely abolishes spine loss in APP transgenic cultures and does not affect controls $(n=12)$. $\left({ }^{\#} p<0.05\right.$ and ${ }^{\# \#} p<0.01$ indicate a significant decrease and ${ }^{+} p<0.05 ;{ }^{++} p<0.001$ a significant increase compared with untreated cultures; mean $\pm \mathrm{SEM}$; one-tailed unpaired Student's $t$ test). mush., Mushroom spine; stub., stubby spine; str.rad., stratum radiatum. Scale bars, $5 \mu \mathrm{m}$. region was chosen, since generally more neurons were infected in this region (Fig. $1 A$, left) and since previous results indicated that the CA3 region was more susceptible to tau-mediated degeneration than the CA1 region (Shahani et al., 2006). For evaluation, same regions were imaged at days 2, 3, and 4 postinfection (Fig. 

EGFP-tau
APP-
transgenic

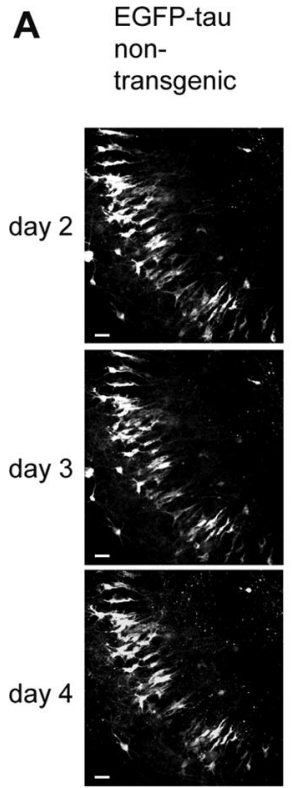

$$
\begin{aligned}
& \text { EGFP-tau } \\
& \text { APP- } \\
& \text { transgenic }
\end{aligned}
$$
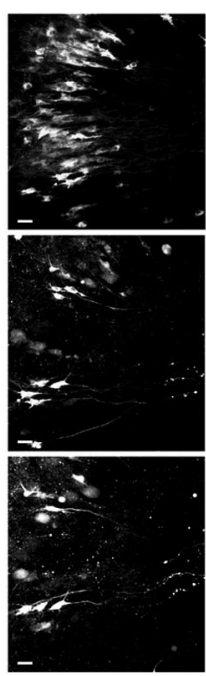

$\square$ non transgenic

B

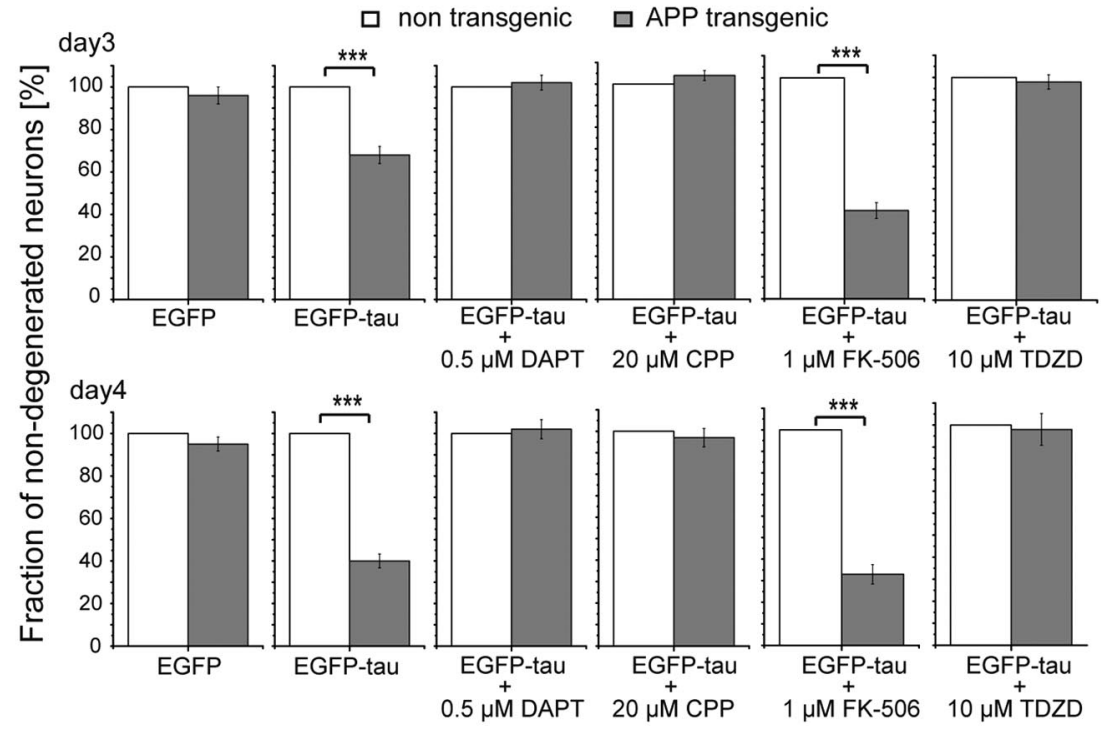

C

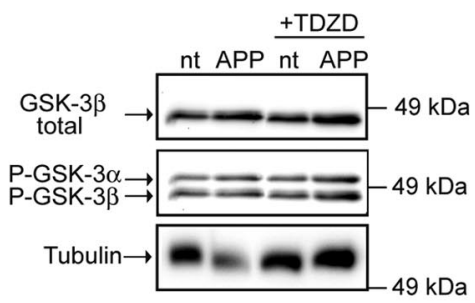

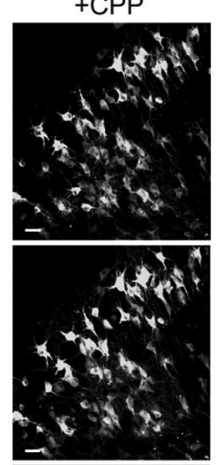

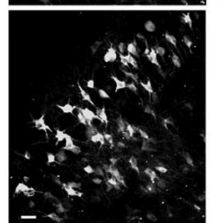

EGFP-tau

APP-

transgenic + FK-506
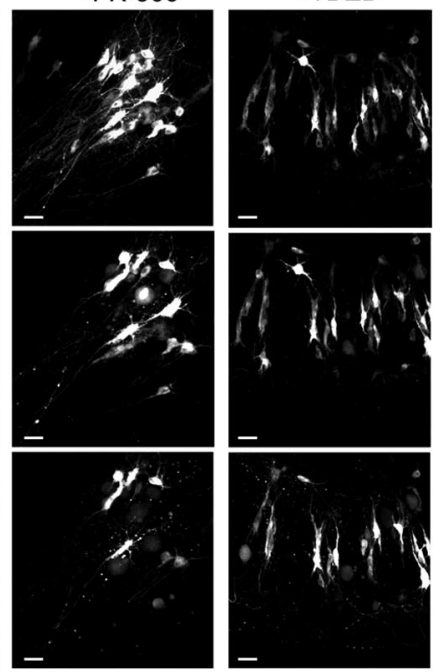

$4 A$ ) and intact neurons according to morphological criteria were counted (see Materials and Methods for details). After expression of EGFP-wt tau in nontransgenic cultures, most neurons survived (Fig. $4 A$, left). In contrast, a massive degeneration of neurons was observed after EGFP-tau expression on APP transgenic background. Degeneration was evident by a complete loss of neurons or the development of a ballooned phenotype. Quantification revealed a progressive loss of neurons from $30 \%$ (day 3 ) to $60 \%$ (day 4 ) $(p<0.001)$ (Fig. $4 B$ ). In contrast, no difference was observed between APP transgenic and nontransgenic cultures after expression of EGFP alone. Preventing A $\beta$ formation by treatment with the $\gamma$-secretase inhibitor DAPT abolished tau toxicity in APP transgenic cultures, indicating that the presence of $A \beta$ is responsible for induction of tau toxicity. To determine whether NMDAR, calcineurin, and GSK-3 $\beta$ activation is also involved in $\mathrm{A} \beta$-induced tau toxicity, cultures were treated with the respective inhibitors. CPP and TDZD treatment abolished neuronal loss, whereas FK-506 had no effect (Fig. 4A,B).

To specify the effect of $A \beta$ on GSK $-3 \beta$, Western blots were performed to determine the amounts of total and phosphorylated (inactive) GSK-3 $\beta$ in lysates of hippocampal slices (Fig. 4C, left). In APP transgenic cultures the expression level of GSK-3 $\beta$ was significantly increased by $\sim 80 \%$ (Fig. $4 C$, top right). Application of TDZD caused a significant increase in phosphorylated (inactive) GSK-3 $\beta$ on APP transgenic background by $\sim 70 \%$ (Fig. 4C, bottom right). The data suggest that TDZD reduces the amount of active GSK-3 $\beta$, which is produced by the increased expression in APP transgenic cultures. Together, the data indicate that neurodegeneration is induced by $\mathrm{A} \beta$ and that $\mathrm{A} \beta$ requires tau. Tau toxicity is induced by a cascade involving NMDARs and GSK-3 $\beta$ activation but not calcineurin. The fact that NMDAR blockage prevents neurodegeneration makes it unlikely that AICD is involved in the pathologic processes in our system.

Figure 4. Effect of CPP, FK-506, and TDZD on the survival of wt tau-expressing neurons in the CA3 region of hippocampal slice cultures. $A$, Live imaging of EGFP-tau-expressing CA3 neurons from nontransgenic and APP transgenic cultures from day 2 to day 4 postinfection after treatment as indicated. Scale bars, $25 \mu \mathrm{m}$. B, Quantification of cell loss on day 3 (top) and day 4 (bottom) postinfection standardized to the respective nontransgenic control. The fraction of nondegenerated neurons as determined by morphological criteria is shown. No difference in cell survival between APP transgenic and nontransgenic cultures expressing only EGFP is observed. EGFP-tau expression results in progressive loss of neurons in cultures from APP transgenic mice. Treatment with $\gamma$-secretase inhibitor DAPT, NMDAR antagonist CPP, or GSK-3 $\beta$ inhibitor TDZD but not calcineurin inhibitor FK- 506 abolishes tau-dependent neuronal loss on APP transgenic background. C, Effect of TDZD on expression and phosphorylation of GSK-3 $\beta$ in APP and nontransgenic slices as determined by Western blot analysis (left). Quantification of total GSK-3 $\beta$ relative to tubulin (top right) and of phospho-GSK-3 $\beta$ relative to total GSK-3 $\beta$ (bottom, right). Expression of GSK-3 $\beta$ is increased in APP transgenic slices. TDZD treatment increases phospho-GSK-3 $\beta$ (inactive GSK-3 $\beta$ ) levels. The experiment was performed in triplicate. Values are $\leftarrow$

shown as mean \pm SEM $(\boldsymbol{B})$ and mean $\pm S D(C)$ with ${ }^{*} p<$ $0.05,{ }^{* * *} p<0.001$; Student's $t$ test $[n=11$ (EGFP), $n=8$ (EGFP-tau, nontransgenic), $n=12$ (EGFP-tau, APP transgenic), $n=8$ (DAPT, nontransgenic), $n=12$ (DAPT, APP transgenic), $n=9$ (FK-506, nontransgenic), $n=10$ (FK-506, APP transgenic), $n=9$ (TDZD)]. 


\section{Disease-relevant tau mutants}

differentially induce cell death in

combination with $\mathbf{A} \boldsymbol{\beta}$

It has been shown that overexpression of FTDP-17 tau mutants such as P301L and R406W in transgenic mice leads to the development of NFTs and neuronal degeneration (Lewis et al., 2000; Zhang et al., 2004). The accumulated tau was phosphorylated at disease-relevant residues (Ikeda et al., 2005). Combination with APP or A $\beta$ increased tangle formation in P301L mice (Lewis et al., 2000; Götz et al., 2001). To analyze the effect of tau phosphorylation and tau mutations in combination with $\mathrm{A} \beta$, EGFP-tagged PHP tau, a less phosphorylatable tau construct (Ala tau), and the two FDTP-17 mutants P301L and R406W tau were prepared in Sindbis virus (Fig. 5A). To determine the effect of the APP transgene on phosphorylation of the different tau mutants, slices from APP transgenic and nontransgenic mice were infected with the constructs and quantitative Western blot analysis was performed. Detection used the PHF-1 antibody that reacts with a phosphorylated epitope at S396 and S404, which is also phosphorylated by GSK-3 $\beta$ (Shahani and Brandt, 2002). Phosphorylation of wt tau at the PHF-1 site was significantly increased by $\sim 25 \%$ on APP transgenic background compared with nontransgenic control indicating that $A \beta$ caused increased phosphorylation of wt tau (Fig. $5 B$ ). Compared with wt tau, both FTDP-17 mutants showed a drastically reduced phosphorylation on nontransgenic background at the PHF- 1 site by 65 and $45 \%$ (R406W tau and P301L, respectively). More importantly, $\mathrm{A} \beta$ did not affect the phosphorylation level of both mutants, suggesting that $A \beta$ differentially affects phosphorylation of wt tau and FTDP-17 mutants. As expected, PHP tau and Ala tau were not immunoreactive with PHF-1, since the epitope had been mutated to glutamate and alanine, respectively.

Survival of neurons in the CA3 region expressing tau mutants was determined by live imaging (Fig. 5C). Expression of PHP tau on a nontransgenic background resulted in a progressive loss of neurons compared with wt tau ( $40 \%$ at day 3 and $50 \%$ at day 4 ). In contrast to wt tau, loss of neurons was not increased after expression of PHP tau on an APP background. Expression of the less phosphorylatable Ala tau construct did not induce cell loss on either nontransgenic or APP transgenic background. This indicates that increased phosphorylation as mimicked by our pseudohyperphosphorylated con-
A
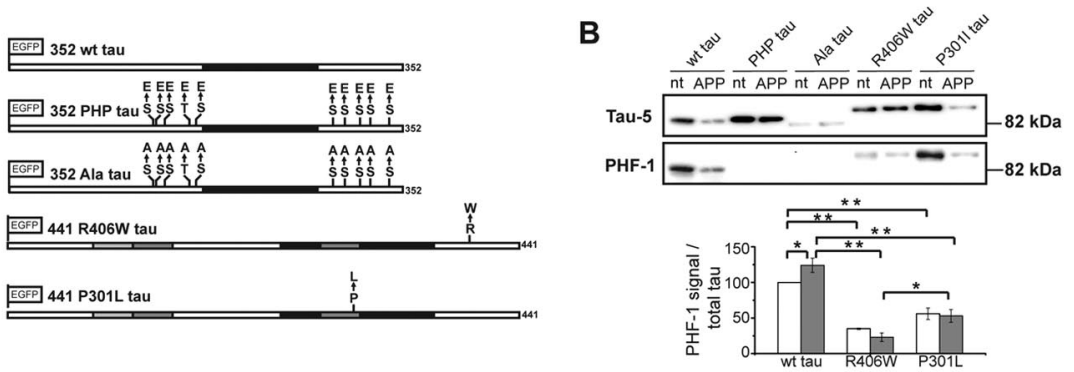

C
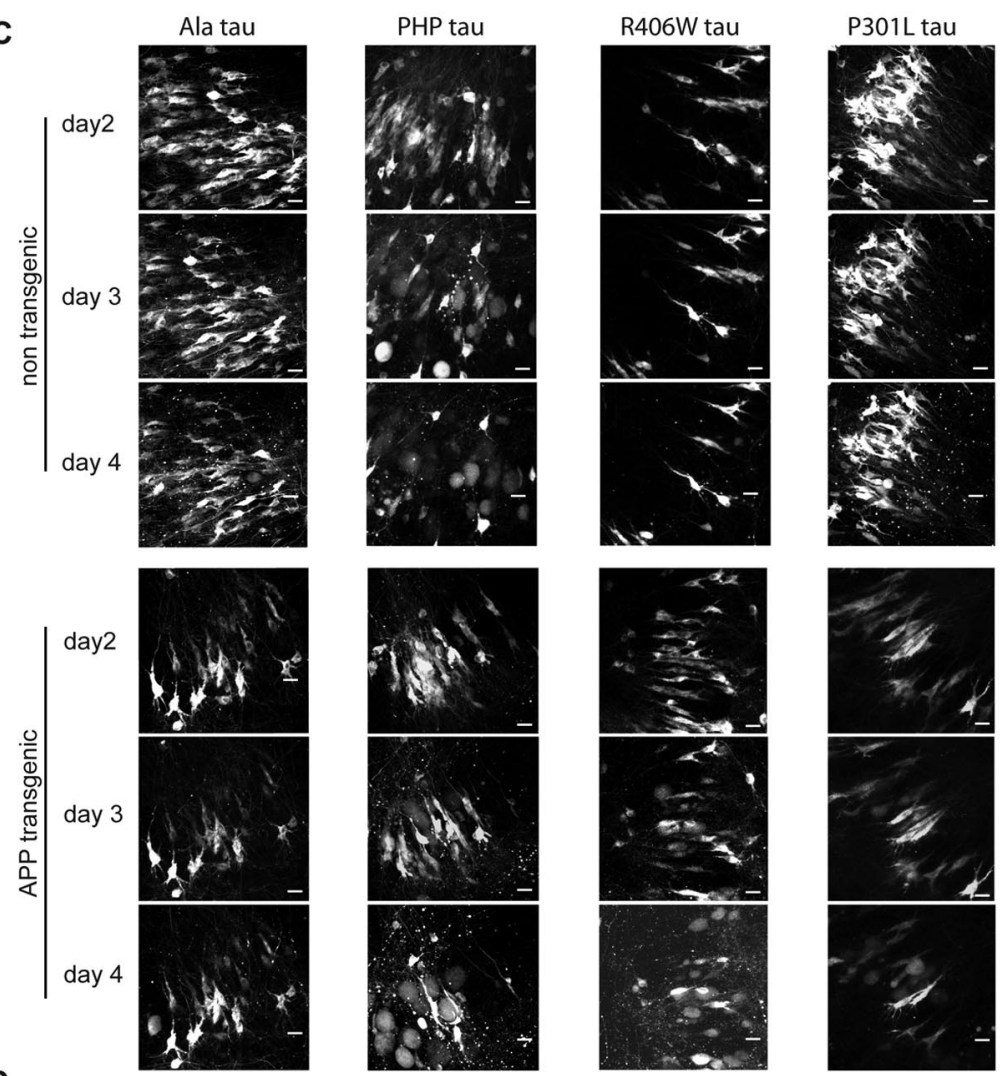

day 3

$\square$ non transgenic

$\square$ APP transgenic

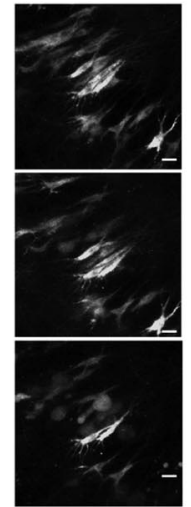

day 4
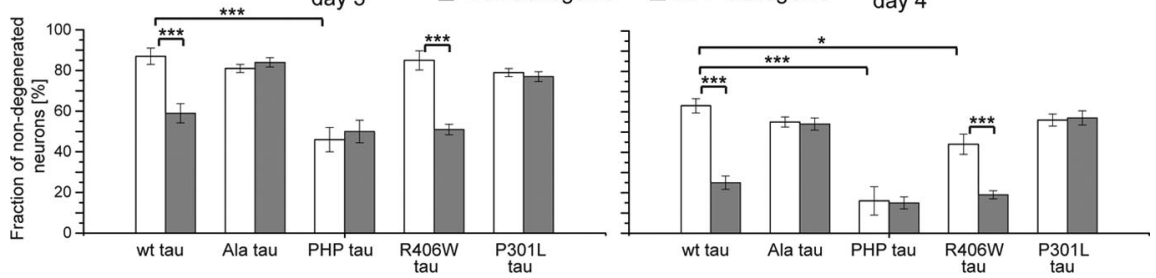

Figure 5. Survival of hippocampal CA3 neurons after expression of disease-relevant tau constructs in hippocampal slice cultures. $\boldsymbol{A}$, Schematic representation of the primary structure of the used tau constructs. $\boldsymbol{B}$, Western blot showing expression of the different tau constructs (Tau-5) and phosphorylation at the PHF- 1 site. Quantification of PHF-1 signal relative to total tau shows increased phosphorylation of wt tau on APP transgenic background. Phosphorylation of R406W tau and P301L tau is reduced compared with wt tau by 65 and 44\%, respectively. In contrast to wt tau, phosphorylation of R406W tau and P301L tau is not increased on APP background. Expression levels of the different constructs varied due to different numbers of infected cells. Experiment was performed in triplicate. $\boldsymbol{C}$, Live imaging of hippocampal CA3 neurons from nontransgenic (top) or APP transgenic mice (bottom) expressing EGFP-tagged tau mutants from day 2 to day 4 postinfection. Scale bars, $25 \mu \mathrm{m}$. D, Quantification of cell loss on day 3 (left) and day 4 (right). Cell numbers on days 3 and 4 are shown relative to day 2 (set as 100\%) for the respective construct. Strong and progressive cell death is seen for cells expressing PHP tau, independent of transgenic background. Expression of R406W tau causes increased neuronal loss in nontransgenic controls on day 4 compared with wt tau expression and strongly induces cell death in APP transgenic cultures. No difference is seen in Ala tau- and P301L-expressing neurons in APP transgenic cultures and nontransgenic controls. Values are shown as mean \pm SD $(\boldsymbol{B})$ and mean \pm SEM $(\boldsymbol{D})$ with ${ }^{*} p<0.05$ and ${ }^{* * *} p<0.001$; Student's $t$ test $[n=8$ (wt tau, nontransgenic), $n=12$ (wt tau, APP transgenic), $n=8$ (Ala tau), $n=14$ (PHP tau, nontransgenic), $n=$ 12 (PHP tau, APP transgenic), $n=8$ (R406W tau), $n=13$ (P301L tau, nontransgenic), $n=10$ (P301L tau, APP transgenic)]. 
A $\begin{array}{cc}\text { EGFP- } & \text { EGFP- } \\ \text { R406W tau } & \text { R406W tau } \\ \text { non transgenic } & \text { APP transgenic }\end{array}$
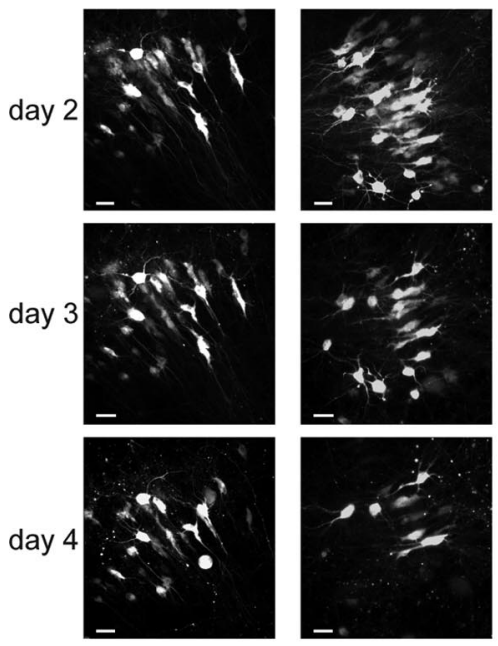

$\square$ non transgenic

B

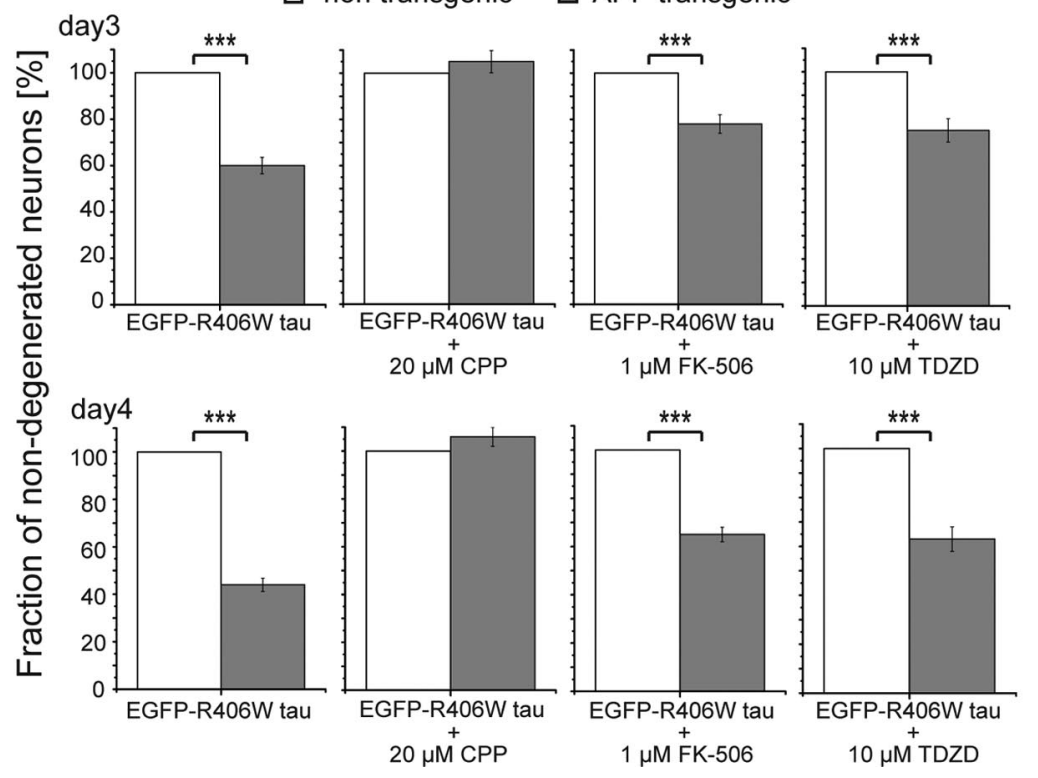

Figure 6. Effect of CPP, FK-506, and TDZD on the survival of R406W tau-expressing neurons in the CA3 region of hippocampal slice cultures. $A$, Live imaging of EGFP-R406W tau-expressing CA3 neurons from nontransgenic and APP transgenic cultures from day 2 to day 4 postinfection after treatment as indicated. Scale bars, $25 \mu \mathrm{m}$. B, Quantification of cell loss on day 3 (top) and day 4 (bottom) postinfection standardized to the respective nontransgenic control. The fraction of nondegenerated neurons as determined by morphological criteria is shown. Neuronal loss is increased after expression of R406W tau on APP transgenic background compared with nontransgenic control. This effect is abolished by treating cultures with CPP but not with FK-506 or TDZD. Note that treatment with FK-506 and TDZD also decreased neuronal survival of the controls. All values are shown as mean \pm SEM with ${ }^{* * *} p<0.001$; one-tailed unpaired Student's $\mathrm{t}$ test; $[n=8$ (R406W tau, untreated), $n=9$ (R406W tau, nontransgenic, (PP), $n=$ 12 (R406W tau, APP transgenic, (PP), $n=8$ (R406W tau, nontransgenic, FK-506), $n=9$ (R406W tau, APP transgenic, FK-506), $n=9$ (R406W tau, TDZD)].

struct is required for the toxic properties of tau in the presence of $\mathrm{A} \beta$. Interestingly, expression of the two FTDP-17 mutants, P301L and R406W tau, differentially affected the survival of neurons dependent on the presence of $\mathrm{A} \beta$. While neuronal death was significantly increased after expression of R406W tau on an APP background ( $p<0.001$ ), no change was observed after expression of P301L. In addition, significant neuron loss was observed at day 4 with $\mathrm{R} 406 \mathrm{~W}$ tau (20\% loss compared with wt tau; $p=$ 0.01 ) but not with P301L tau in nontransgenic controls (Fig. $5 D$ ).
Thus, although both mutations induce a tauopathy in patients, the mechanism by which they affect neuronal survival appears to differ, as evidenced by their differential effect in the presence and absence of $\mathrm{A} \beta$. To control for similar expression levels of the different constructs, the amounts of wt tau, R406W tau, and P301L tau in single neurons were determined by measuring the fluorescence intensity of neurons after infection with the respective EGFP-tau construct. Three days postinfection mean fluorescence intensities of $101 \pm 10$ and $101 \pm 9 \%$ for R406W tau and P301L tau, respectively, were observed in nontransgenic controls (wt tau set to $100 \% ; n=10$ per construct). In APP transgenic slices, fluorescence intensities of $97 \pm 7,96 \pm 10$, and $101 \pm 9 \%$ were observed for wt tau, R406W tau, and P301L tau, respectively. The data confirm that the differential effects are caused by different toxic properties of the respective tau constructs rather than by different expression levels.

The data indicate that phosphorylation of wt tau is required for $A \beta$-induced cell death. Mimicking high phosphorylation using PHP tau abolishes the requirement for $A \beta$. The FTDP-17 tau mutant R406W shows some neurotoxicity by itself. Interestingly, the increased toxicity of R406W tau in APP transgenic slices is not paralleled by increased phosphorylation at the PHF-1 site, suggesting that the mechanisms by which $\mathrm{A} \beta$ confers toxicity to tau are different for wt tau and R406W tau.

\section{$A \beta$ increases R406W tau toxicity by a pathway involving NMDARs but independent of GSK-3 $\beta$ or calcineurin} Our data suggest that $A \beta$ affects wt tau and the FTDP-17 mutant R406W tau by different mechanisms. To determine the signal transduction pathway involved in $\mathrm{A} \beta$-induced R406W tau toxicity, cell survival was analyzed for CA3 neurons expressing R406W tau in APP transgenic or nontransgenic cultures treated with $\mathrm{CPP}$, FK-506, or TDZD (Fig. 6A). Blocking NMDAR activity with CPP abolished $\mathrm{A} \beta$ induced toxicity of R406W tau, whereas FK-506 treatment failed to show a protective effect. This indicates that both wt tau and R406W tau toxicity is mediated by NMDARs but not calcineurin. In contrast, treatment with TDZD did not affect cell survival on an APP transgenic background in R406W tau-expressing cells, while it was protective in wt tau-expressing neurons (compare Figs. $6 B$, right, $4 B$, right). This suggests that GSK- $3 \beta$ is not involved in $\mathrm{A} \beta$-induced $\mathrm{R} 406 \mathrm{~W}$ tau toxicity. Thus, the data indicate that $\mathrm{A} \beta$ induces R406W tau toxicity by a different pathway than it does for wt tau. 


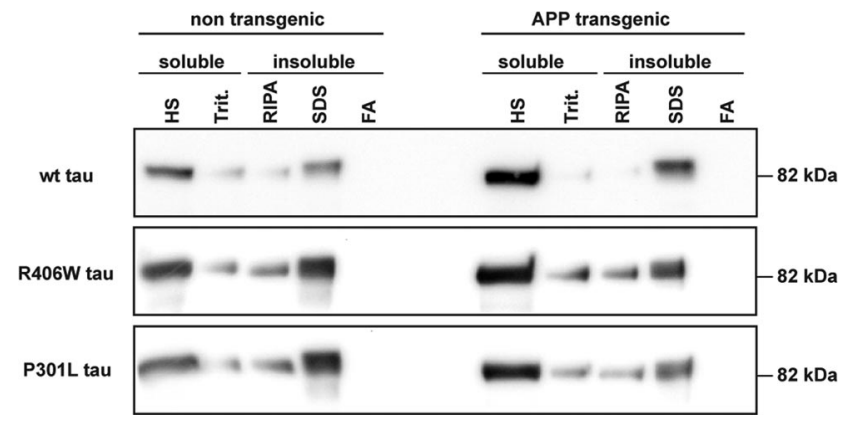

Figure 7. Sequential extraction of wt, R406W, and P301L tau from hippocampal slice cultures. Tau solubility profiles from lysates of infected nontransgenic and APP transgenic slices. The extraction was performed using the following buffers of increasing stringency: high salt (HS), 1\% Triton (Trit.), RIPA, 2\% SDS, and 70\% FA. The majority of wt tau protein was found in the HS fraction. In nontransgenic controls, R406W tau and P301L tau is increased in the insoluble fraction to $54-57 \%$ compared with wt tau (42\%). On the APP transgenic background the insoluble tau fraction is decreased for all constructs (33-39\%). Equal amounts of lysates from each extraction step were loaded and stained with Tau- 5 antibody against total tau.

\section{The solubility profiles of wt, R406W, and P301L tau do not correlate with toxicity}

To determine whether the toxicity of the different tau constructs correlates with potential tau aggregation, the solubility profiles of wt tau, R406W tau, and P301L tau were analyzed by a sequential extraction protocol using buffers of increasing stringency (Fig. $7)$. With all constructs and at every condition, tau was not detected in the FA fraction, implicating the absence of highly insoluble tau species. Compared with wt tau, both FTDP-17 mutants showed a slightly reduced solubility on a nontransgenic background (wt tau, 58\%; R406W tau, 45\%; P301L tau, 42\% in the soluble fraction), which was mainly evident by an increased amount of tau in the SDS fraction. Interestingly, on an APP transgenic background, solubility of all constructs was increased by $10 \%$ for wt tau and by 16 and 22\% for R406W tau and P301L tau, respectively. Thus, R406W tau and P301L tau do not show differences in their aggregation propensities, although they strongly differ in their toxic properties in the presence and absence of $A \beta$ as described above (Fig. 5). This indicates that the solubility of wt tau, R406W tau, and P301L tau does not correlate with toxicity in our experiments.

\section{Discussion}

We have established an ex vivo model of $\mathrm{AD}$ using organotypic hippocampal slice cultures from $\mathrm{APP}_{\mathrm{SDL}}$ transgenic mice in combination with Sindbis virus-mediated expression of fluorescent labeled tau constructs. Detailed spine analysis was performed by algorithm-based evaluation of high-resolution confocal images of dendritic segments of CA1 and CA3 pyramidal neurons. Neuronal survival was determined by live imaging. This approach permits analysis of the relation between cell death and synaptic changes and the interaction between $A \beta$ and tau pathology. In addition, it permits determination of signal transduction mechanisms that are involved in each of these pathways in an experimentally well accessible system.

Previously it has been shown that senile plaques and $\mathrm{A} \beta$ oligomers reduce spine density in vivo and in slice cultures (Tackenberg et al., 2009). In agreement with this, we observed that spine density was strongly reduced in $\mathrm{APP}_{\mathrm{SDL}}$ transgenic cultures. Interestingly, spines were not affected by tau expression. Spine loss was abolished in the presence of the $\gamma$-secretase inhibitor DAPT, suggesting that the effect on spines was due to A $\beta$. Since $\mathrm{APP}_{\mathrm{SDL}}$ mice do not develop plaques before the age of 18 months

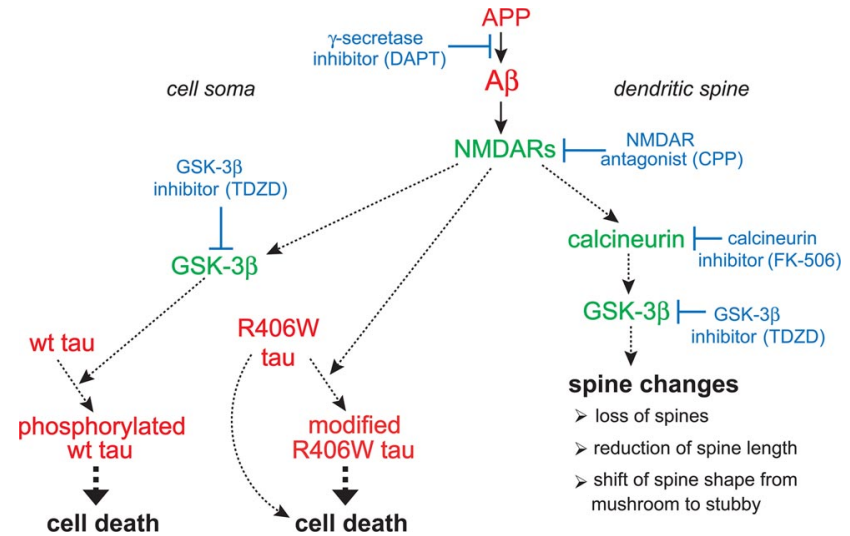

Figure 8. Schematic representation showing the proposed pathways that mediate spine pathology and tau-dependent cell death. The formation of $A \beta$ is central, since blocking $A \beta$ production by treatment with the $\gamma$-secretase inhibitor DAPT abolishes spine changes and the induction of tau toxicity. NMDAR activity is required for both induction of spine alterations and $A \beta$-induced tau toxicity, since blocking NMDAR activity with CPP abolishes both pathologies. $A \beta$ but not tau causes loss of spines, reduction of spine length, and alterations in spine shape, as evidenced by a shift from mushroom to stubby spines. In contrast, $A \beta$ alone is not neurotoxic but requires tau to induce cell death. GSK-3 $\beta$ is activated by $A \beta$ and participates in both cascades but calcineurin is operating only in mediating spine changes. Within spines, calcineurin is upstream of GSK-3 $\beta$, since calcineurin inhibition mimics the effect of the NMDAR antagonist CPP. In the soma, $A \beta$ activates GSK-3 $\beta$ independent of calcineurin, which is essential for the induction of wt tau toxicity, since blocking GSK-3 $\beta$ activity abolishes cell death caused by wt tau in APP transgenic cultures, whereas calcineurin inhibition has no effect on cell survival. In contrast, induction of R406W tau toxicity by $A \beta$ is GSK- $3 \beta$ independent, suggesting that $A \beta$ affects R406W tau by a different mechanism. Continuous lines show direct effects, and dashed lines show indirect effects with potential intermediate steps.

(Blanchard et al., 2003), spine loss was induced by soluble A $\beta$ in our experiments. It was previously reported that addition of soluble $\mathrm{A} \beta$ to slice cultures resulted in increased spine length but did not affect spine head diameter (Shrestha et al., 2006). However, a detailed analysis of changes in spine types caused by $\mathrm{A} \beta$ had not been performed. In nontransgenic cultures, algorithm-based calculation revealed $\sim 50 \%$ mushroom, $40 \%$ stubby, and $10 \%$ thin spines. These numbers closely reflect the distribution in similar cultures that have been evaluated manually by measurements of spine length and head diameter (Zagrebelsky et al., 2005). We observed a reduced percentage of mushroom spines and an increase in the fraction of stubby spines in neurons from APP transgenic mice. The change in spine shape was also observed in dendritic subregions in which no spine loss occurred. This indicates a transition from mushroom to stubby spines but no selective loss of mushroom spines. The morphological change is associated with a reduction in mean spine length, suggesting that $\mathrm{A} \beta$ causes spine retraction. Interestingly, spine volume was not affected. Staining of presynaptic boutons against synaptophysin showed that mushroom and stubby spines from nontransgenic and APP transgenic animals were in close apposition with synaptophysin dots. While this is suggestive for the presence of functional synapses also after the change in spine shape it cannot be excluded that the strength of synaptic transmission is affected.

Cell survival was determined by live imaging of infected CA3 pyramidal neurons. We did not observe a difference between nontransgenic and $\mathrm{APP} \mathrm{SDL}_{\mathrm{S}}$ transgenic cultures after expression of EGFP indicating that $A \beta$ alone is not neurotoxic. In contrast, we observed massive neurodegeneration after expression of EGFP-wt tau in APP transgenic cultures compared with nontransgenic controls, which was prevented by DAPT. This indicates that tau is essential for $\mathrm{A} \beta$-induced neurodegeneration, which is in agreement 
with studies using dissociated hippocampal cultures (Rapoport et al., 2002). This raises the question by which mechanism $A \beta$ induces tau toxicity. We showed that phosphorylation of wt tau is increased on APP transgenic background at the PHF-1 site (phosphorylated S396 and S404), an epitope that is among others phosphorylated by GSK-3 $\beta$ (Shahani and Brandt, 2002). In agreement, expression levels of GSK-3 $\beta$ were increased in APP transgenic cultures. Increased expression or activation of several tau kinases including GSK-3 $\beta$ has already been reported in $A D$ (Blurton-Jones and Laferla, 2006). Mutation of 10 of the major phosphorylation sites to alanine to prevent phosphorylation abolished increased toxicity in the presence of $\mathrm{A} \beta$. In turn, PHP tau in which the same sites were mutated to glutamate to mimic a permanent hyperphosphorylation showed toxicity even on a nontransgenic background supporting that increased phosphorylation at disease-relevant sites can confer toxicity to tau.

Many mouse models for AD have been developed by expressing tau with FTDP-17 mutations in combination with mutated APP or PS1. However, no FAD cases were reported in which tau is mutated. Thus, it is important to compare the effect of $\mathrm{A} \beta$ on wt tau and FTDP-17 tau mutants to determine whether these provide a valid model for $\mathrm{AD}$ pathology. We determined the behavior of two FTDP-17 mutants that have been frequently used. We observed that R406W tau is more toxic than wt tau on a nontransgenic background and becomes highly toxic in the presence of $\mathrm{A} \beta$. In contrast, P301L tau did not show any toxicity neither in nontransgenic controls nor on an APP background. In contrast to wt tau, the increased toxicity of R406W tau in APP transgenic slices is not paralleled by increased phosphorylation at the PHF-1 site, suggesting that the mechanism how A $\beta$ confers toxicity to tau is different for wt tau and R406W tau. This makes it questionable to use FTDP-17 tau mutants in combination with $\mathrm{APP}$ or $\mathrm{A} \beta$ as a model for neurodegeneration in $\mathrm{AD}$.

It is unknown whether soluble or aggregated tau causes neurodegeneration. We determined the aggregation propensity of wt tau, R406W tau, and P301L tau in the presence or absence of A $\beta$. Interestingly, $A \beta$ increased the solubility of all constructs. R406W tau and P301L tau did not show differences in their aggregation propensity although they strongly differ in their toxic properties in the presence and absence of $A \beta$. Thus, our data indicate that the solubility of wt tau, R406W tau, and P301L tau does not correlate with toxicity and suggests that tau-dependent neurodegeneration occurs in the absence of major tau aggregates. This is in agreement with the observation that tau-dependent neurodegeneration occurred in a Drosophila model without NFT formation (Wittmann et al., 2001) and that in a zebrafish model degeneration preceded tangle formation (Paquet et al., 2009). In a mouse model, neuron number stabilized and memory recovered after tau suppression despite continuous accumulation of NFTs (Santacruz et al., 2005).

Our approach permits to investigate the signal transduction pathways involved in mediating both spine changes and cell death (Fig. 8). We show that blocking A $\beta$ production by treatment with the $\gamma$-secretase inhibitor DAPT abolished both spine changes and the induction of tau toxicity. This indicates that the generation of $\mathrm{A} \beta$ is upstream of both processes, which supports the amyloid cascade hypothesis. It has been shown previously that soluble $\mathrm{A} \beta$ oligomers can bind to or near NMDARs (Lacor et al., 2007). Shankar et al. (2007) have shown that blockade of NMDARs abolished spine loss, which had been induced by the acute addition of soluble $A \beta$. A $\beta$ induced LTD via activation of calcineurin and GSK-3 $\beta$ (Li et al., 2009). This raises the question whether NMDARs, calcineurin, and GSK-3 $\beta$ are also involved in mediating spine changes in $\mathrm{APP}_{\mathrm{SDL}}$ cultures. We found that treatment with NMDAR antagonist CPP, calcineurin inhibitor FK506 , and GSK-3 $\beta$ inhibitor TDZD abolished A $\beta$-mediated spine loss. Since the effect of FK-506 closely resembled the effect of CPP including a reduction of spine number in controls, GSK-3 $\beta$ appears to be downstream of calcineurin, which is consistent with the finding that GSK-3 $\beta$ activity is regulated, among others, by calcineurin (Lee et al., 2005). Thus, $A \beta$ induces a cascade involving NMDAR, calcineurin, and GSK- $3 \beta$ activation, which alters neuronal connectivity as observed in $\mathrm{AD}$. It has been suggested that $\mathrm{A} \beta$ influences spines by mimicking an LTD-like partial blockade of NMDARs followed by activation of cofilin and calcineurin which finally leads to degradation of the actin cytoskeleton within the spine (Shankar et al., 2007). A complete blockade of NMDAR activity by NMDAR antagonist CPP may therefore prevent the induction of downstream cascades and protect against $\mathrm{A} \beta$-induced spine alterations.

We could show for the first time that the induction of tau toxicity by $\mathrm{A} \beta$ is also NMDAR dependent since blockade of NMDARs abolished cell death mediated by wt tau or R406W tau in APP transgenic cultures. In contrast to the cascade causing spine loss, the induction of tau toxicity is not calcineurin dependent. Blocking GSK-3 $\beta$ prevented toxicity of wt tau but not R406W tau on APP transgenic background. This is in agreement with our finding that phosphorylation of R406W tau is not increased at the PHF-1 site in APP transgenic cultures and further supports that wt tau and R406W tau are differentially affected by $\mathrm{A} \beta$.

Although both spine alterations and cell death are mediated by NMDARs, the downstream cascades substantially differ, since a blockade of calcineurin prevented spine loss but not cell death. We hypothesize that both cascades occur in different cellular compartments. A $\beta$ may cause spine changes by inducing a cascade involving NMDARs, calcineurin, and GSK-3 $\beta$ within the spine itself while inducing a calcineurin-independent cascade involving NMDARs and GSK3 $\beta$ at the soma, which causes wt tau phosphorylation and cell death.

\section{References}

Blanchard V, Moussaoui S, Czech C, Touchet N, Bonici B, Planche M, Canton T, Jedidi I, Gohin M, Wirths O, Bayer TA, Langui D, Duyckaerts C, Tremp G, Pradier L (2003) Time sequence of maturation of dystrophic neurites associated with Abeta deposits in APP/PS1 transgenic mice. Exp Neurol 184:247-263.

Blurton-Jones M, Laferla FM (2006) Pathways by which Abeta facilitates tau pathology. Curr Alzheimer Res 3:437-448.

Bourne J, Harris KM (2007) Do thin spines learn to be mushroom spines that remember? Curr Opin Neurobiol 17:381-386.

Braak H, Braak E (1991) Neuropathological stageing of Alzheimer-related changes. Acta Neuropathol 82:239-259.

Chen P, Gu Z, Liu W, Yan Z (2007) Glycogen synthase kinase 3 regulates $\mathrm{N}$-methyl-D-aspartate receptor channel trafficking and function in cortical neurons. Mol Pharmacol 72:40-51.

Dovey HF, John V, Anderson JP, Chen LZ, de Saint Andrieu P, Fang LY, Freedman SB, Folmer B, Goldbach E, Holsztynska EJ, Hu KL, JohnsonWood KL, Kennedy SL, Kholodenko D, Knops JE, Latimer LH, Lee M, Liao Z, Lieberburg IM, Motter RN, et al. (2001) Functional gammasecretase inhibitors reduce beta-amyloid peptide levels in brain. J Neurochem 76:173-181.

Ehrengruber MU, Lundstrom K, Schweitzer C, Heuss C, Schlesinger S, Gähwiler BH (1999) Recombinant Semliki Forest virus and Sindbis virus efficiently infect neurons in hippocampal slice cultures. Proc Natl Acad Sci U S A 96:7041-7046.

Eidenmüller J, Fath T, Maas T, Pool M, Sontag E, Brandt R (2001) Phosphorylation-mimicking glutamate clusters in the proline-rich region are sufficient to simulate the functional deficiencies of hyperphosphorylated tau protein. Biochem J 357:759-767. 
Fath T, Eidenmüller J, Brandt R (2002) Tau-mediated cytotoxicity in a pseudohyperphosphorylation model of Alzheimer's disease. J Neurosci 22:9733-9741.

Ferreira A, Lu Q, Orecchio L, Kosik KS (1997) Selective phosphorylation of adult tau isoforms in mature hippocampal neurons exposed to fibrillar A $\beta$. Mol Cell Neurosci 9:220-234.

Götz J, Chen F, van Dorpe J, Nitsch RM (2001) Formation of neurofibrillary tangles in P301l tau transgenic mice induced by Abeta 42 fibrils. Science 293:1491-1495.

Haass C, Selkoe DJ (2007) Soluble protein oligomers in neurodegeneration: lessons from the Alzheimer's amyloid beta-peptide. Nat Rev Mol Cell Biol 8:101-112.

Hardy J, Selkoe DJ (2002) The amyloid hypothesis of Alzheimer's disease: progress and problems on the road to therapeutics. Science 297:353-356.

Ikeda M, Shoji M, Kawarai T, Kawarabayashi T, Matsubara E, Murakami T, Sasaki A, Tomidokoro Y, Ikarashi Y, Kuribara H, Ishiguro K, Hasegawa M, Yen SH, Chishti MA, Harigaya Y, Abe K, Okamoto K, St GeorgeHyslop P, Westaway D (2005) Accumulation of filamentous tau in the cerebral cortex of human tau R406W transgenic mice. Am J Pathol 166:521-531.

Koh IY, Lindquist WB, Zito K, Nimchinsky EA, Svoboda K (2002) An image analysis algorithm for dendritic spines. Neural Comput 14:1283-1310.

Lacor PN, Buniel MC, Furlow PW, Clemente AS, Velasco PT, Wood M, Viola KL, Klein WL (2007) A $\beta$ oligomer-induced aberrations in synapse composition, shape, and density provide a molecular basis for loss of connectivity in Alzheimer's disease. J Neurosci 27:796-807.

Lee YI, Seo M, Kim Y, Kim SY, Kang UG, Kim YS, Juhnn YS (2005) Membrane depolarization induces the undulating phosphorylation/dephosphorylation of glycogen synthase kinase 3beta, and this dephosphorylation involves protein phosphatases $2 \mathrm{~A}$ and $2 \mathrm{~B}$ in SH-SY5Y human neuroblastoma cells. J Biol Chem 280:22044-22052.

Leschik J, Welzel A, Weissmann C, Eckert A, Brandt R (2007) Inverse and distinct modulation of tau-dependent neurodegeneration by presenilin 1 and amyloid-beta in cultured cortical neurons: evidence that tau phosphorylation is the limiting factor in amyloid-beta-induced cell death. J Neurochem 101:1303-1315.

Lewis J, McGowan E, Rockwood J, Melrose H, Nacharaju P, Van Slegtenhorst M, Gwinn-Hardy K, Paul Murphy M, Baker M, Yu X, Duff K, Hardy J, Corral A, Lin WL, Yen SH, Dickson DW, Davies P, Hutton M (2000) Neurofibrillary tangles, amyotrophy and progressive motor disturbance in mice expressing mutant (P301L) tau protein. Nat Genet 25:402-405.

Li S, Hong S, Shepardson NE, Walsh DM, Shankar GM, Selkoe D (2009) Soluble oligomers of amyloid Beta protein facilitate hippocampal longterm depression by disrupting neuronal glutamate uptake. Neuron 62:788-801.

Miyasaka T, Ding Z, Gengyo-Ando K, Oue M, Yamaguchi H, Mitani S, Ihara Y (2005) Progressive neurodegeneration in C. elegans model of tauopathy. Neurobiol Dis 20:372-383.

Mulkey RM, Endo S, Shenolikar S, Malenka RC (1994) Involvement of a calcineurin/inhibitor-1 phosphatase cascade in hippocampal long-term depression. Nature 369:486-488.

Paquet D, Bhat R, Sydow A, Mandelkow EM, Berg S, Hellberg S, Fälting J,
Distel M, Köster RW, Schmid B, Haass C (2009) A zebrafish model of tauopathy allows in vivo imaging of neuronal cell death and drug evaluation. J Clin Invest 119:1382-1395.

Peineau S, Taghibiglou C, Bradley C, Wong TP, Liu L, Lu J, Lo E, Wu D, Saule E, Bouschet T, Matthews P, Isaac JT, Bortolotto ZA, Wang YT, Collingridge GL (2007) LTP inhibits LTD in the hippocampus via regulation of GSK3beta. Neuron 53:703-717.

Peters A, Kaiserman-Abramof IR (1970) The small pyramidal neuron of the rat cerebral cortex. The perikaryon, dendrites and spines. Am J Anat 17:321-355.

Rapoport M, Dawson HN, Binder LI, Vitek MP, Ferreira A (2002) Tau is essential to beta-amyloid-induced neurotoxicity. Proc Natl Acad Sci U S A 99:6364-6369.

Santacruz K, Lewis J, Spires T, Paulson J, Kotilinek L, Ingelsson M, Guimaraes A, DeTure M, Ramsden M, McGowan E, Forster C, Yue M, Orne J, Janus C, Mariash A, Kuskowski M, Hyman B, Hutton M, Ashe KH (2005) Tau suppression in a neurodegenerative mouse model improves memory function. Science 309:476-481.

Shahani N, Brandt R (2002) Functions and malfunctions of the tau proteins. Cell Mol Life Sci 59:1668-1680.

Shahani N, Subramaniam S, Wolf T, Tackenberg C, Brandt R (2006) Tau aggregation and progressive neuronal degeneration in the absence of changes in spine density and morphology after targeted expression of Alzheimer's disease-relevant tau constructs in organotypic hippocampal slices. J Neurosci 31:6103-6114.

Shankar GM, Bloodgood BL, Townsend M, Walsh DM, Selkoe DJ, Sabatini BL (2007) Natural oligomers of the Alzheimer amyloid- $\beta$ protein induce reversible synapse loss by modulating an NMDA-type glutamate receptor-dependent signaling pathway. J Neurosci 27:2866-2875.

Shrestha BR, Vitolo OV, Joshi P, Lordkipanidze T, Shelanski M, Dunaevsky A (2006) Amyloid beta peptide adversely affects spine number and motility in hippocampal neurons. Mol Cell Neurosci 33:274-282.

Steiner H, Capell A, Leimer U, Haass C (1999) Genes and mechanisms involved in beta-amyloid generation and Alzheimer's disease. Eur Arch Psychiatry Clin Neurosci 249:266-270.

Stoppini L, Buchs PA, Muller D (1991) A simple method for organotypic cultures of nervous tissue. J Neurosci Methods 37:173-182.

Tackenberg C, Ghori A, Brandt R (2009) Thin, stubby or mushroom: spine pathology in Alzheimer's disease. Curr Alzheimer Res 6:261-268.

Wittmann CW, Wszolek MF, Shulman JM, Salvaterra PM, Lewis J, Hutton M, Feany MB (2001) Tauopathy in Drosophila: neurodegeneration without neurofibrillary tangles. Science 293:711-714.

Zagrebelsky M, Holz A, Dechant G, Barde YA, Bonhoeffer T, Korte M (2005) The p75 neurotrophin receptor negatively modulates dendrite complexity and spine density in hippocampal neurons. J Neurosci 25:9989-9999.

Zhang B, Higuchi M, Yoshiyama Y, Ishihara T, Forman MS, Martinez D, Joyce S, Trojanowski JQ, Lee VM (2004) Retarded axonal transport of R406W mutant tau in transgenic mice with a neurodegenerative tauopathy. J Neurosci 24:4657-4667.

Zheng WH, Bastianetto S, Mennicken F, Ma W, Kar S (2002) Amyloid beta peptide induces tau phosphorylation and loss of cholinergic neurons in rat primary septal cultures. Neuroscience 115:201-211. 